\title{
Convergence of forepaw somatosensory and motor cortical projections in the striatum, claustrum, thalamus, and pontine nuclei of cats
}

Jared Brent Smith ( $\square$ jasmith@salk.edu )

Salk Institute for Biological Studies https://orcid.org/0000-0002-0273-4898

Shubhodeep Chakrabarti

Penn State College of Medicine

Todd M. Mowery

Rutgers Robert Wood Johnson Medical School

Kevin D. Alloway

Penn State College of Medicine

Original Article

Keywords: motor cortex, somatosensory cortex, striatum, claustrum, thalamus, pons

Posted Date: April 9th, 2021

DOI: https://doi.org/10.21203/rs.3.rs-194390/v1

License: (c) (i) This work is licensed under a Creative Commons Attribution 4.0 International License.

Read Full License

Version of Record: A version of this preprint was published at Brain Structure and Function on October 19th, 2021. See the published version at https://doi.org/10.1007/s00429-021-02405-6. 


\section{Abstract}

The basal ganglia and pontocerebellar systems regulate somesthetic-guided motor behaviors, and receive prominent inputs from sensorimotor cortex. Additionally, the claustrum and thalamus are forebrain subcortical structures that have connections with somatosensory and motor cortices. Our previous studies in rats have shown that primary and secondary somatosensory cortex (S1 and S2) send overlapping projections to the neostriatum and pontine nuclei, whereas overlap of primary motor cortex (M1) and S1 was much weaker. Additionally, we have shown that M1, but not S1, projects to the claustrum in rats. The goal of the current study was to compare these rodent projection patterns with connections in cats, a mammalian species that evolved in a separate phylogenetic superorder. Three different anterograde tracers were injected into the physiologically identified forepaw representations of M1, S1, and S2 in cats. Labeled fibers terminated throughout the ipsilateral striatum (caudate and putamen), claustrum, thalamus, and pontine nuclei. Digital reconstructions of tracer labeling allowed us to quantify both the normalized distribution of labeling in each subcortical area from each tracer injection, as well as the amount of tracer overlap. Surprisingly, in contrast to our previous findings in rodents, we observed M1 and S1 projections converging prominently in striatum and pons, whereas S1 and S2 overlap was much weaker. Furthermore, whereas rat S1 does not project to claustrum, we confirmed dense claustral inputs from S1 in cats. These findings suggest that the basal ganglia, claustrum, and pontocerebellar systems in rat and cat have evolved distinct patterns of sensorimotor cortical convergence.

\section{Introduction}

The cerebral cortex contains numerous modality-specific regions, with each area specialized for a specific type of information processing (for review see Krubitzer 2009). For example, somatosensory and motor cortical areas are thought to process aspects of touch and movement, respectively (Borich et al. 2015; Chapin and Lin 1984; Hall and Lindholm 1974; Hoffer et al. 2003; Kaas 2004; but see Matyas et al. 2010). However, information across these two modalities must be integrated in both directions to achieve appropriate somesthetic-guided movements and accurate sensory perceptions of the resulting bodily motion (Arce-McShane et al. 2016; Khateb et al. 2017; Lee et al. 2008, 2013; Murthy and Fetz 1992; PaiVieira et al 2013; Umeda et al. 2019, Zagha et al. 2013). In addition to direct cortico-cortical connections between sensory and motor cortices that mediate such integration, each of these cortical areas also projects to numerous subcortical structures, some of which represent additional opportunities for sensorimotor integration (Battaglia-Mayer and Caminiti 2019; Chakrabarti et al. 2006, 2008; Colechio and Alloway 2009; Fabri and Burton 1991; Ghosh et al. 1987; Hamadjida et al. 2016; Hoffer et al. 2003, 2005; Huerta and Pons 1990; Izraeli and Porter 1995; Jones et al. 1978; Mao et al. 2011; Smith and Alloway 2013; Stepniewska et al. 1993, 2020; Suter and Shepherd 2015; Vogt and Pandya 1977).

Among the subcortical targets of sensorimotor cortex, the thalamus contains higher-order nuclei such as the posteromedial nucleus (POm), which receives convergent inputs from both sensory and motor cortical areas, as well as projecting back to both areas (for review see Alloway 2008; Alloway et al. 2008; Hoffer 
and Alloway 2001; Lu and Lin 1993; Mo and Sherman 2019). In addition, the claustrum has connections that could facilitate interactions between sensory and motor cortices (for review see Smith et al. 2019a; Jackson et al. 2020). Our previous work in rodents, for example, has shown that the claustrum receives dense inputs from motor cortex and sends bifurcating projections back to both sensory and motor cortex (Smith and Alloway 2010, 2014; Smith et al. 2012).

In addition to cortical projections to the thalamus and claustrum, the cerebral cortex also sends topographically organized projections to the basal ganglia and pontocerebellar systems (Brodal 1978; Dudman and Gerfen 2015; Flaherty and Graybiel 1991; Hoover and Strick 1993; Kunzle 1975, 1977; Oh et al. 2014; Proville et al. 2014; Schmahmann and Pandya 1997; Schwarz and Mock 2001; Suzuki et al. 2012; Wiesendanger and Wiesendanger 1982a,b). In turn, both the basal ganglia and the cerebellum provide feedback to sensorimotor cortex via multi-synaptic outputs to the thalamus, which are believed to remain segregated, thus forming parallel cortico-subcortico re-entrant loops (Alexander et al. 1986, 1990; Aoki et al. 2019a; Brodal and Bjaalie 1992; Hoover and Strick 1993; Mercier et al. 1990; Middleton and Strick 2000; Parent and Hazrati 1995; but see Aoki et al. 2019b for evidence of convergence in basal ganglia loops). However, though largely topographic, whole-cortex mapping studies of cortico-striatal projections have demonstrated convergence of modality related cortical inputs into functional domains of striatum that are thought to have specific behavioral relevance (Hinitryan et al. 2016; Hunnicutt et al. 2016; Oh et al. 2014). In the sensorimotor domain, previous work in rodents and primates has demonstrated convergence of sensory and motor cortices in the dorsolateral region of the striatum (Alloway et al. 2006; Charpier et al. 2020; Flaherty and Graybiel 1993, 1995; Hoffer et al. 2001; Hooks et al. 2018; Hoover et al. 2003; Lee et al. 2019; Ramanathan et al. 2002), an area of the striatum that is responsible for guiding sensorimotor habits (see Alloway et al. 2017 for review). Similar anatomical tracing studies in the cortico-pontine system of rodents and primates indicate parallel themes of integration to those observed in the cortico-striatal system (Bjaalie and Brodal 1989; Leergaard et al. 2000a,b; Leergaard et al. 2004; Legg et al. 1989; Schwarz and Thier 1995; Wiesendanger et al. 1972; Wiesendanger and Wiesendanger 1982a,b).

In the current work, we sought to expand on these previous studies by investigating cortical sensorimotor integration in multiple subcortical structures in felines. The feline clade, which is part of the Laurasiatheria superorder, diverged from rodents and primates, members of the superorder Euarchontoglires, nearly 100 million years ago (Murphy et al. 2004). By employing the same physiologically guided, neuroanatomical tracing paradigm used previously to map sensorimotor convergence in rodents (Alloway et al. 2000; Hoffer and Alloway 2001; Leergaard et al. 2004), we can make direct comparisons to felines to assess the hypothesis that different phylogenetic clades evolved distinct neural circuits for sensorimotor integration. Therefore, we used microstimulation mapping of primary motor cortex (M1) and receptive field mapping of primary and secondary somatosensory cortex (S1 and S2, respectively) to identify overlapping forepaw representations in each cortical area. We then injected each area with a different neuroanatomical tracer. This triple-tracer paradigm allowed us to assess integration from three modality related cortical areas in the striatum, claustrum, thalamus, and basal pontine nuclei within the same animal. Our results show that whereas S1 and S2 projections show 
strong convergence in rodents, felines show significantly more S1-M1 integration, indicating that different strategies in cortico-subcortical interactions evolved across phylogenetically separate lineages.

\section{Material And Methods}

Anatomical tracing experiments were performed on ten adult male felines (Charles River) weighing 3.1$5.0 \mathrm{~kg}$. All procedures were approved by Penn State University's Institutional Animal Care and Use Committee and conformed to National Institute of Health standards.

\section{Animal surgery}

Surgical procedures were performed using sterile technique with support from veterinary staff for anesthesia and animal care (Penn State College of Medicine, Department of Comparative Medicine). In preparation for injections, animals received an injection of dexamethasone $(0.1 \mathrm{mg} / \mathrm{kg}$, intramuscular) the day before surgery. The following morning, animals were initially anesthetized with an intra-muscular injection of ketamine $(10 \mathrm{mg} / \mathrm{kg})$ and acepromazine $(0.5 \mathrm{mg} / \mathrm{kg})$. The femoral artery was catheterized, the head was shaved, the trachea was intubated through the oral cavity, and the femoral artery was catheterized to enable intravenous injections of dexamethasone $0.1 \mathrm{mg} / \mathrm{kg}$ ) and chloramphenicol $(50 \mathrm{mg} / \mathrm{kg}$ ). The head was then fixed into a stereotaxic device (Kopf) and the animal was ventilated with a mixture of oxygen and sevoflurane (1-2\%) to maintain a stable anesthetic plane for the remainder of the procedure as determined by lack of nociceptive withdrawal reflexes and flaccid muscle tone. A sensor was attached to the tongue and vital signs (heart rate, specific oxygen, respiratory rate, and in/end tidal $\mathrm{CO}_{2}$ ) were monitored continuously (Surgivet) and recorded every fifteen minutes. Fluids were maintained via saline drip connected to the femoral catheter. Body temperature was monitored via a rectal thermistor and was maintained by hydraulic heat pumps underneath the animal, as well as a hot air enclosure around the torso (Bear-Hugger). Ophthalmic ointment was applied to the eyes to prevent corneal drying.

The skin over the cranium was disinfected with betadine and a sterile drape was place over the entire head. Prior to the incision, the skin over the area of the craniotomies was infiltrated with numerous subcutaneous injections of lidocaine to provide local anesthesia. A single large incision was made over the right side of the skull to provide access to the frontal and parietal bones. The skin was retracted, and the underlying fascia and muscle was resected to expose bregma and the sites of the craniotomies. After measuring with surgical rulers, two craniotomies approximately $15 \mathrm{~mm}$ in diameter were drilled, with one spanning the primary motor (M1) and primary somatosensory (S1) cortices, and the other around the secondary somatosensory (S2) cortex.

\section{Intracranial microstimulation (ICMS)}

Following craniotomies, tungsten microelectrodes (FHC, 0.5-1.0M $\Omega$ ), affixed to a stereotaxic micromanipulator were inserted in $\mathrm{M} 1$ coordinates used in previous mapping studies (Alloway and Burton 1985a,b for S1 and S2). The electrode was inserted into the cortex via a small slit in the dura made with a $27 \mathrm{G}$ needle held by a pair of forceps. The electrode was lowered to a depth of $\sim 1.0-1.5 \mathrm{~mm}$ below the 
dural surface to target layer 5, which contains corticospinal neurons (Suter and Shepherd, 2015). A constant current source (BAK BSI-2) controlled by a digital timer (Master-8), was used to administer electrical stimulation ( $0.7 \mathrm{~ms}$ pulse, $250 \mathrm{~Hz}$ frequency). Current levels were initially high $(\sim 100 \mu \mathrm{A})$ and reduced to identify the minimum current necessary for eliciting limb or forepaw movements at each cortical site (typically $<30 \mu \mathrm{A}$ ). To evoke muscle twitches, sevoflurane levels had to be reduced to $0.5-$ $0.75 \%$; this led to a slight increase in muscle-tone but maintained insensitivity to forepaw pinches. The electrode was moved to numerous sites to obtain a detailed map of the body representation of $M 1$, to identify the center of the forepaw representation. Typical movements included dorsoflexion of the entire forepaw and brief twitches of individual digits.

\section{Extracellular neuronal recordings.}

In order to identify the forepaw representation in S1 cortex and S2 cortex, a tungsten microelectrode (FHC, 1.0-1.5M $\Omega$ ) was used to map both areas. Initial coordinates were identified based on previous studies (Alloway and Burton 1985 a,b; Felleman et al. 1983), and detailed maps were obtained for each individual animal. Electrodes were inserted via a dural slit and lowered to a depth of $400-600 \mu \mathrm{m}$ below the surface, corresponding to layer 4 , the primary input layer from thalamus. Neuronal activity was amplified (Dagan, $1 \mathrm{k}$ head-stage amp, $2 \mathrm{k}$ amp, band pass filtered $300 \mathrm{~Hz}-5 \mathrm{kHz}$ ) and subsequently observed on an oscilloscope (Grass Digital) as well as through an audio monitor. The wooden end of a cotton-tipped applicator was used to probe the surface of the skin to elicit a neural response. Receptive fields were drawn, and coordinates recorded to provide a detailed map of both S1 cortex and S2 cortex, such that injections could be targeted to overlapping representations of the forepaw.

\section{Tracer injections.}

Following detailed mapping of M1, S1, and S2 cortices, tracer injections were targeted to overlapping representations of the forelimb. Each cortical area was targeted with a different anterograde tracer (Molecular Probes, Eugene OR) including Fluoro-Ruby (FR, D-1817), Alexa-Fluor 488 (AF, D-2290), and Biotinylated Dextran Amine (BDA, D-1956). While BDA was always injected into M1, FR and AF injections were injected into either S1 or S2 (see Table 1 for summary). Tracers were injected by means of $2.0 \mu \mathrm{L}$ Hamilton syringes secured onto a microinjector (Kopf) attached to the stereotaxic frame as done previously (Alloway et al., 2000, 2009). Each syringe had a pulled glass pipette, with a 10-50 $\mu \mathrm{m}$ tip, cemented onto the end of the Hamilton syringe needle to minimize the damage to the cortex. The tip of the pipette was inserted $\sim 1.7 \mathrm{~mm}$ from the surface to insure tracer deposit within layer 6 and in more superficial layers as the tracer tended to seep upward along the injection pipette. Tracers were injected slowly $(100 \mathrm{~nL} / \mathrm{min})$, and total tracer volume injected at each cortical site varied from 400 to $900 \mathrm{nl}$. Each cortical area (ie., M1, S1, or S2) received 4-7 injections to cover a wide region of the forepaw representation. 
Table 1

Summary of tracer injections in cats.

\begin{tabular}{|llll|}
\hline Animal ID & M1 Tracer Injection & S1 Tracer Injection & S2 Tracer Injection \\
\hline TI-2 & BDA & FR & AF \\
\hline TI-3 & BDA & FR & AF \\
\hline TI-8 & BDA & FR & AF \\
\hline TI-9 & BDA & AF & FR \\
\hline TI-10 & BDA & FR & AF \\
\hline Anterograde tracers: BDA - biotinylated dextran amine; FR - FluoroRuby; AF- AlexaFluor488 dextran \\
\hline
\end{tabular}

Following mapping and tracer injections, Gel-film was placed over the cortical surface and Gel-foam inserted into the craniotomy. The incision was subsequently sutured and triple antibiotic ointment applied to the wound margins. The margins of the skin incision were injected with bupivacaine $(1-2 \mathrm{ml})$ to provide local anesthesia, and the animal received a long-lasting analgesic, buprenorphine $(0.01 \mathrm{mg} / \mathrm{kg}$ $I M)$, just prior to discontinuation of the sevoflurane and removal of the head from the stereotaxic. Upon recovery of self-respiration, the intubation tube and femoral catheter were removed, and animals were monitored until fully conscious and able to right themselves. Animals were returned to their home cage and monitored daily with veterinary-assisted post-operative care for the duration of the experiment.

\section{Histology.}

After 10 days to allow for tracer transport, animals were deeply anesthetized with ketamine, xylazine and acepromazine. A femoral catheter was inserted, and animals were over-dosed with a lethal quantity of sodium pentobarbital (Nembutal $100 \mathrm{mg} / \mathrm{kg}$ ). Animals were trans-cardially perfused with heparinized saline (3 cc heparin/L saline) until the liver blanched, and then subsequently perfused with $4 \%$ paraformaldehyde in $0.1 \mathrm{M}$ PBS and $4 \%$ paraformaldehyde in $0.1 \mathrm{M}$ PBS with $10 \%$ sucrose. The brain and spinal cord were removed and stored in $4 \%$ paraformaldehyde in $0.1 \mathrm{M}$ PBS with $30 \%$ sucrose for cryoprotection. The day after the perfusion, the arachnoid was removed from the cortical surface with Dumont forceps and the brain and spinal cord were blocked.

After thorough fixation of the brain ( 3 days or until it sank in the fixative solution), the brain was cut in $60-\mu \mathrm{m}$ sections on a freezing microtome. Sections were stored in 0.1M PBS and refrigerated. For processing, tissue was split into 5 serially-ordered groups for: (1) Nissl staining, (2) BDA processing, (3) fluorescence-only, and two back-up sets. Nickel-enhanced BDA processing was performed as previously described (Hoffer et al. 2005). Briefly, sections were agitated in $0.3 \% \mathrm{H}_{2} \mathrm{O}_{2}$ and then washed with $0.3 \%$ Triton X-100 (pH = 7.4) in 0.1M PBS before a 2-hour incubation in an activated avidin-biotin horseradish peroxidase solution (Vector Novocastra Laboratories, Burlingame, $\mathrm{CA}$ ). Sections were rinsed in $0.1 \mathrm{M}$ PB and then incubated in a solution of $0.05 \%$ diaminobenzidine (DAB), $0.0005 \% \mathrm{H}_{2} \mathrm{O}_{2}$, and $0.04 \% \mathrm{NiCl} 2$ in 
$0.1 \mathrm{M}$ Tris buffer $(\mathrm{pH}=7.1)$ for $9-12$ minutes. The reaction was terminated with washes in a $0.1 \mathrm{M} P B$ solution. After processing, sections were mounted on gel-coated glass slides, dried overnight, then dehydrated with ethanol, defatted with xylene and finally cover-slipped with Cyto-seal.

\section{Anatomical analysis.}

Tracer labeling was analyzed with an Olympus $\mathrm{BH}-2$ microscope fitted with 1x, 2x, 4x, 10x, and 20x objectives and 10x eyepieces. BDA and Nissl stains were observed using brightfield, whereas FR and AF fluorescence was imaged using a combined fluorescein isothiocyanate/tetrarhodamine isothiocyanate (FITC/TRITC) filter cube (51004v2, Chroma Technology, Rockingham, VT). Labeled neuronal soma and labeled axonal varicosities of terminal arborizations were digitally reconstructed with respect to anatomical landmarks using a computer controlled AccuStage reconstruction system (St Paul, MN) and stored to hard drive for subsequent analysis. For anterogradely-labeled fibers, synaptic boutons were plotted under 20x magnification, as these structures represent putative synapses (Kincaid and Wilson 1996).

To quantify the convergence of projections from M1, S1, and S2 cortices, the plotted reconstructions of terminal labeling were analyzed using custom software (Hoffer et al., 2005) or a custom module in MD Plot (AccuStage software), which imposed a grid of $50 \mu \mathrm{m} \times 50 \mu \mathrm{m}$ squares onto the sections. This bin size was chosen to allow comparison to previous rat data obtained in our lab (Alloway et al. 2000; Hoffer et al. 2001, 2003, 2005; Leergaard et al. 2004). For each tracer, bins containing at least two boutons were counted as labeled and colored appropriately (BDA-blue, FR-red, AF, green). Bins labeled by two tracers were colored white so that tracer overlap is expressed as a percentage of all colored bins (ie., red, green blue, and white) that were labeled as white.

Digital photomicrographs of tracer-labeling and Nissl staining were acquired by a Cool Snap HQ CCD digital camera (Retiga) mounted on the BH-2 microscope. Images were stored as TIF files and imported into our graphic design software (Deneba Systems, Canvas X, Miami, FL) to generate figures. Quantification of tracer labeling was analyzed in Microsoft Excel, with statistics and graphing performed using Graphpad Prism.

\section{Results}

Neuronal tracers were administered to ten cats (Charles River). Cases were included in the final analysis only if the histological examination confirmed that all three tracer injections were present and spanned both the supragranular and infragranular layers of cortex because virtually all cortical layers send projections to one or more of the subcortical regions that we analyzed in the present study (Royce 1982; Levesque et al. 1992; Mao et al. 2011). Furthermore, we only analyzed cases in which transported tracer labeling was apparent in all subcortical regions of interest: striatum, claustrum, thalamus, and the basal pontine nuclei. Five of the experiments satisfied these criteria and were included in the final analysis (see Table 1 for summary of injections). Representative photomicrographs of tracer injections from case TI-8, shown in Fig. 1, depict biotinylated dextran amine (BDA) injected into the forepaw region of primary 
motor cortex (M1; Fig. 1C, $\mathrm{C}^{\prime}$ ), Fluororuby (FR) injected into the forepaw representation of primary somatosensory cortex (S1; Fig. 1d,d'), and Alexa-fluor 488 (AF) injected into the forepaw representation of secondary somatosensory cortex (S2; Fig. 1e,e'). All of these sites were identified physiologically, either via intracranial microstimulation (ICMS) mapping of M1 cortex or receptive field mapping of the S1 and S2 cortical areas, which are demonstrated in Fig. 1b. Additionally, the Nissl-stained sections shown in Fig. 1 show hallmarks of motor and sensory cortex. In Fig. 1c, Nissl staining of the M1 injection site reveals characteristics of agranular cortex (i.e. motor cortex), which lacks a layer 4, but shows an expanded layer 5 with large cell bodies that likely represent corticofugal projections targeting the spinal cord and other brainstem structures. In contrast, Nissl staining in Fig. 1d and 1e of S1 and S2 reveals a prominent granular layer 4 , which is characteristic of sensory cortex. The injection sites in each of these regions span all layers of cortex, including layers 3,5 and 6 , which represent the output layers to the subcortical regions of interest. The other four cases included in the final analysis showed similar injection characteristics.

In addition to the physiologic mapping, and histological verification of the injection sites, we also examined tracer labeling in thalamus to confirm the functional placement of the tracer injections. Figure 2 shows representative tracer labeling in the thalamus from the injection sites shown in Fig. 1 for case TI-8. As demonstrated by the digital reconstruction in Fig. $2 \mathrm{~b}$ and the photomicrograph in Fig. $2 \mathrm{c}$ tracer labeling appeared in the ventromedial (VM) nucleus, a motor related region of thalamus, following tracer injections in M1 cortex. Anterogradely labeled terminals and retrogradely labeled neuronal cell bodies were observed in the ventrobasal complex following the S1 and S2 tracer injections (Fig. 2d-e). The presence of anterogradely labeled terminals further indicates that our tracer injections infiltrated layers 5 and 6 as these layers represent the origin of most corticothalamic projection neurons. Interestingly, the labeling in the thalamus was markedly segregated, with the bulk of labeling from each tracer injection largely forming its own topographic domain. Segregated thalamic domains were also observed in another representative case (case TI-9 shown below), in which BDA was injected in M1, AF in $\mathrm{S} 1$, and FR in S2.

\section{Cortico-striatal projections.}

Previous studies in rodents and primates, have reported that all sensorimotor cortical areas send topographically-organized projections to the striatum (Aoki et al. 2019; Brodal 1978; Dudman and Gerfen 2015; Flaherty and Graybiel 1991, 1993, 1995; Hinitryan et al. 2016; Hoover and Strick 1993; Hunnicutt et al. 2016; Kunzle, 1975, 1977; Oh et al. 2014). Consistent with these previous findings, we observed tracer labeling from M1, S1, and S2 throughout the dorsolateral caudate and the putamen. The digital reconstruction shown in Fig. 3 from case TI-8 reveals dense intermingling of S1, S2, and M1 projections to a band of neuropil along the dorsolateral edge of the caudate that stretched $3 \mathrm{~mm}$ along the anteriorposterior axis. Overlap analysis of the tracer labeling in a grid of $50-\mu \mathrm{m}^{2}$ bins revealed strong overlap of tracer labeling from M1 and S1 injections (shown as white bins in Fig. 4a), whereas M1-S2 and S1-S2 overlap was minimal. In this representative case, cortico-striatal labeling from the AF injection in S2 was relatively caudal and noticeably weaker than the labeling from the M1 and S1 injections, which could 
account for the low overlap with projections from S2 cortex. In another case, however, we varied which tracers were injected in the cortical areas, and we still observed minimal M1-S2 and S1-S2 overlap. As shown below, in case TI-9 where FR was injected in S2, we found that the labeled projections from S2 cortex terminated in zones that were interdigitated with the overlapping terminal labeling resulting from the $\mathrm{M} 1$ and $\mathrm{S} 1$ tracer injections.

\section{Cortico-claustral projections.}

Analysis of the tracer labeling in the claustrum revealed anterogradely labeled terminals and retrogradely labeled neuronal cell bodies throughout a $1 \mathrm{~mm}$ anterior-posterior extent of the dorsolateral claustrum (shown for case TI-8 in Fig. 5a). Like the striatum, we observed marked overlap between M1 and S1 projections, as shown in the tracer overlap analysis in Fig. 5b-d. Projections from S2 terminated more medially in an adjacent region to the area of M1-S1 terminals and did not overlap with any of the corticoclaustral projections from S1 and M1. A similar pattern can be seen in the digital reconstruction from case TI-9 shown below.

\section{Cortico-pontine projections.}

Analysis of the tracer labeling in the pontine nuclei revealed anterogradely labeled terminals throughout a $\sim 3 \mathrm{~mm}$ anterior-posterior extent of the structure, positioned on both the medial and lateral sides of the descending pyramidal tract white matter (shown for case TI-8 in Fig. 6a). As in the striatum, we observed substantial overlap between M1 and S1 projections, as shown in the tracer overlap analysis in Fig. 6b-d. In contrast to the striatum, however, we observed stronger input from S2 cortex, which showed moderate overlap with both $\mathrm{M} 1$ and $\mathrm{S} 1$ projections.

\section{Quantitative analysis.}

The amount of anterograde tracer labeling can vary due to differences in tracer volume, efficiency and detectability. To mitigate any such affects, we varied which tracer was injected into each cortical area. Figure 7 shows a representative example (case TI-9), where the most efficient tracer, FR, was injected into S2 cortex (BDA was injected into M1, and AF into S1). As shown throughout Fig. 7, the pattern of tracer labeling was identical to what was observed in the representative example (TI-8) shown in earlier figures.

To further determine whether these factors could affect our overlap analysis we performed statistical analysis on the total numbers of terminals counted across all animals used in the final analysis $(n=5)$. The total number of counts summed across all subcortical structures (e.g. striatum, claustrum, thalamus, and pons) was found to vary for each tracer (BDA: $11517 \pm 3534$; FR: 22057 \pm 7841 ; AF: $2625 \pm 880$ ). However, though some tracers produced more labeled terminals than others (e.g. FR $>A F$ ), statistical analysis did not reveal any significant difference in the total number of labeled varicosities across different tracers (one-way ANOVA, $F=3.80, p=0.053$ ). These results suggest that differences in the amount or efficiency of the different tracers was likely not a significant factor in our overlap analysis. Furthermore, we varied which tracer was injected into what cortical site (see Table 1), further limiting the 
effect of tracer efficiency on the results of our overlap analysis. To further investigate tracer efficiency, we also calculated the total bouton counts produced based on cortical injection site (Fig. 8a, M1: 11517 \pm 3534; S1: $19910 \pm 8598$; S2: $4772 \pm 2623$ ) finding no significant differences (Kolmogorov Smirnoff test for normality, $p>0.10$; one-way ANOVA, $F=1.85, p=0.20$ ). This second analysis further demonstrates that tracer efficiency was not a significant factor in our overlap analysis.

The next quantitative analysis we performed was to identify the relative amount of labeling in each subcortical region of interest from each cortical area injected (i.e. M1, S1, and S2). This distribution was normalized for each tracer within each animal then, percentages were averaged across subjects, thus making these measurements insensitive to tracer efficiency issues. As shown in Fig. 8b, this analysis revealed significant differences in the distribution of projections from these cortical areas to these subcortical structures (2-way ANOVA, interaction $F=43.24, p<0.0001$ ). Post-hoc Bonferroni comparisons showed M1 projections to striatum were significantly stronger than those from $S 1(t=8.11, p<0.001)$ and $S 2(t=8.93, p<0.001)$, whereas in thalamus M1 projections were significantly weaker than those from S1 $(t=9.96, p<0.001)$ and $S 2(t=11.47, p<0.001)$.

Analysis of tracer overlap using $50 \mu \mathrm{m}^{2}$ bins revealed significant differences in the overlap of labeled projections from M1, S1, and S2 (2-way ANOVA, interaction $F=4.00, p<0.01$ ). Post-hoc Bonferroni tests revealed that M1-S1 overlap was significantly greater than M1-S2 or S1-S2 overlap in both the striatum ( $t$ $=5.43, p<0.001$ and $t=5.38, p<0.001$; respectively $)$ and in the pons $(t=3.44, p<0.05$ and $t=2.70$, n.s. $)$. We then compared these data from felines with those from our previous studies in rodents. The injection scheme showing location of anterograde tracer injections in rodents for our previous studies (Leergaard et al. 2004) is shown in Fig. 9c. Quantitative analysis of the overlap analysis (bin size $50 \mu \mathrm{m}^{2}$ ) in the rodent tracing studies shows more S1-S2 overlap than M1-S1, that was significant in the pons $(t=4.87, p$ $<0.001)$. These findings are in opposition to the findings in felines, where S1-M1 overlap was significantly greater, indicating cross-species differences in sensorimotor convergence.

\section{Discussion}

In this study we analyzed the convergence of sensory and motor cortical projections in subcortical structures; specifically, the striatum, claustrum, thalamus and pons. Using a triple anterograde tracer approach in cats, we injected the forepaw representation in M1, S1, and S2 cortices (which are equidistant) and analyzed the distribution and overlap of anterogradely labeled terminals. These new findings were subsequently compared to our previous tracing experiments in rodents (Hoffer and Alloway 2001; Leergaard et al. 2004), to determine how sensorimotor cortical projections to the striatum, claustrum, thalamus and pons vary across evolutionarily distinct lineages (Euarchontoglires and Laurasiatheria).

These experiments yielded several key findings. First, in cats we observed the greatest tracer overlap between $\mathrm{M} 1$ and $\mathrm{S} 1$ terminals in the striatum and pons, whereas in rodents the overlap in these target regions was stronger for the projections from S1 and S2. Second, whereas S1 and S2 cortices in the cat 
sent the majority of their projections to the thalamus ( 70\%), M1 projections to the thalamus were weak $(<20 \%)$; the most abundant projections terminated in the striatum $(\sim 60 \%)$. This finding contrasts with our previous rodent data in which thalamus was consistently the strongest target of M1 (Alloway et al. 2009). Third, in the cat claustrum we observed clear input from both $M 1$ and $S 1$ cortex, in contrast to our previous observations in rats where we observed weak input from M1 forepaw and no input from S1 (Smith and Alloway 2010; Smith et al. 2012). Together these findings suggest evolutionary differences in the principles guiding the distribution and integration of sensorimotor cortical projections to the subcortex.

\section{Relative contribution of sensorimotor projections to subcortical structures}

One of the primary findings of these studies, shown in Fig. 8b, is that while M1 contributed most of its projections to the striatum, S1 and S2 sent the majority of their inputs to the thalamus with equivalently minor contributions to striatum and pons. Furthermore, M1, S1 and S2 projections were almost equally represented within the pons. Finally, all three cortical areas sent relatively few projections to the claustrum compared to striatum, thalamus or pons. These findings may give insight into the relative neural processes that occur in these subcortical structures to guide behavior.

In the case of the claustrum, our findings show relatively few connections with sensorimotor cortex compared to the other subcortical structures. Our previous tracing studies in rodents yielded similar findings (Alloway et al. 2009; Smith and Alloway 2010), Specifically, in rodents we observed that the M1 forepaw region had very sparse connections to the dorsal region of the ipsilateral claustrum, and no input from S1 cortex was detectable (Alloway et al. 2009; Smith et al. 2012). This suggests a limited role of forelimb-related somato-motor inputs in dictating the functional output of the claustrum as discussed in more detail below.

Interestingly, motor cortex in felines contributes very little input to the thalamus compared to S1 and S2 cortices, which sent the overwhelming majority of their inputs to VPL. Cortico-thalamic feedback is thought to play a role in attention by enhancing sensory signals from the periphery and optimizing sensory acuity through receptive field modulation (Alitto and Usrey 2003; Alloway 2008; Briggs and Usrey 2008). Our data suggest that corticothalamic projections play a stronger role in processing sensory information than motor information, at least in felines. Interestingly, in rodents, M1 forepaw projections targeted thalamus (45\%) more strongly than striatum (30\%) (Alloway et al. 2009), providing evidence for species specific patterns in the distribution of cortico-subcortical projections.

The cortico-cerebellar system is thought to be involved in motor planning as well as monitoring and adjusting motor output based on sensory feedback (Chen et al. 2016; Doyon et al. 2003; Gao et al. 2018; Ramnani 2006), whereas striatum is thought to be involved in action selection (Friend and Kravitz 2014; Klaus et al. 2019; Redgrave et al. 1999). By virtue of its dominant inputs to the striatum, our results indicate M1 plays a stronger role in the action selection process than the action refinement process (though it is involved in both). Furthermore, our results reveal substantially more inputs from M1 to the striatum than from $\mathrm{S} 1$ and $\mathrm{S} 2$, suggesting that motor cortex plays a more prominent role in action 
selection than sensory cortex. With regards to the pons, as indicated by the largely equivalent inputs from $\mathrm{M1}, \mathrm{S1}$, and S2, our data indicate an equally important role for motor and sensory information in sensoryguided correction of motor behavior via the cortico-cerebellar system.

\section{Sensorimotor cortico-claustral projections}

The claustrum is a unique subcortical structure whose function remains undefined (for review see Jackson et al. 2020). Many studies have sought to identify its function based on its anatomical connectivity, which is largely organized into reciprocal connections with the neocortex (Smith et al. 2019a, Jackson et al. 2020). One striking finding from our current studies is the minimal representation of sensorimotor inputs to the claustrum, compared to striatum, thalamus and pons. Consistent with other species, these projections were restricted to the dorsolateral region (Smith et al. 2019a), which represents only a small region of the total claustrum. Previous tracing data in cats has suggested that auditory and visual inputs are more prominent in cats (LeVay and Sherk 1981; Macchi et al. 1981). More recent findings in rodents have found the strongest inputs to the claustrum arise from limbic structures like the amygdala, MD thalamus as well as prefrontal cortex (Jackson et al. 2020). Furthermore, the claustrum in rodents has been shown to be a critical node in the rodent homolog of the salience network (Smith et al. $2019 b)$. This potential role in sensory salience is thought to be funneled towards motor cortical areas to direct attention by guiding behavioral output towards the most relevant sensory stimuli. The limited role of somato-motor processing of the forelimb in the cat claustrum, with more expansive auditory and visual inputs, would fit with a role in predation and tracking salient visual and auditory stimuli related to prey. However, additional studies directly comparing these differences is needed to make any definitive conclusions.

In our previous studies mapping the connections of sensorimotor cortex with the claustrum in rodents (Smith and Alloway 2010, 2014; Smith et al. 2012), we observed weak projections from the forepaw representation in M1 cortex to the claustrum and no projections from any region of S1 cortex. In contrast, our current findings in cats clearly revealed dense inputs from both $\mathrm{S} 1$ and $\mathrm{M} 1$ cortices, which terminated in the same overlapping region of the dorsal claustrum, which is the sensorimotor domain in the claustrum of rats and monkeys as well (Smith et al. 2019a). Interestingly, we saw almost no connections between S2 cortex and claustrum in cat, which aligns with an earlier finding observed while mapping claustro-cortical connections in felines using retrograde tracers (Macchi et al. 1981).

These findings further align with other tracing studies across different primate species that have shown differences in cortico-claustral connectivity (Sherk 1986). Specifically, in some primates the primary visual cortex has strong projections to the claustrum, whereas it is absent in other species. Indeed, we have also shown that V1 has no projections to the claustrum in rodents (Smith and Alloway 2014). Interestingly, LeVay and Sherk found that in cats, numerous visual areas, including area 17 contributed profuse projections to the claustrum (LeVay and Sherk 1981). Thus, no clear pattern has yet emerged as to why some sensory cortical areas project to the claustrum and others do not. But, though the functional meaning of these differences is unclear, further systematic study of the cortico-claustral connectome 
across species, and their functional relation to behavioral specializations within those species, will undoubtedly reveal crucial inferences as to the function of the claustrum.

\section{Cortico-striatal and cortico-pontine sensorimotor integration}

It has been well established that the striatum and basal pontine nuclei are organized into functional domains, based largely on studies tracing cortico-striatal and cortico-pontine projections (Brodal 1978; Dudman and Gerfen 2015; Flaherty and Graybiel 1991; Hoover and Strick 1993; Kunzle 1975, 1977; Oh et al. 2014; Schmahmann and Pandya 1997; Wiesendanger and Wiesendanger 1982a,b). Furthermore, functionally related cortical regions, such as sensory and motor cortex, converge onto overlapping areas in both the striatum (Alloway et al. 2006; Charpier et al. 2020; Flaherty and Graybiel 1993, 1995; Hoffer et al. 2001; Hooks et al. 2018; Hoover et al. 2003; Lee et al. 2019; Ramanathan et al. 2002 Stepniewska et al. 2020) and the pons (Bjaalie and Brodal 1989; Leergaard et al. 2000a,b; Leergaard et al. 2004; Legg et al. 1989; Schwarz and Thier 1995; Wiesendanger et al. 1972; Wiesendanger and Wiesendanger 1982a,b). In our current study, we observed M1, S1, and S2 projections terminating in largely overlapping regions of the striatum (both dorsolateral caudate and putamen) and the basal pons. However, closer analysis of the anatomical reconstructions revealed significantly stronger overlap of S1 and M1 projections compared to S1-S2 and M1-S2 projections. This pattern of convergence was comparable for both the striatum and pons, indicating similar rules for sensorimotor integration. However, the functional significance of integrating S1 and M1 information, but not the inputs from S2 is unclear. Additionally, this preference for S1-M1 integration is contrary to our previous findings in rodents, which showed species specific patterns of cortico-subcortical sensorimotor integration.

One possibility for explaining this finding could be if S2 projections are processed in parallel to S1 and thus target different subregions within $\mathrm{M} 1$ and subcortical structures in cats. In our previous tracing studies in rodents (Hoffer and Alloway 2001; Hoffer et al. 2003, 2005; Leergaard et al. 2004; Smith and Alloway 2013), homotypic S1 and S2 whisker representations were found to have overlapping projections in $\mathrm{M} 1$ as well as in other subcortical structures such as striatum and pons. While it has been shown that S2 projects to M1 in cats (Mori 1997), no study has characterized whether S1 and S2 projections overlap in $\mathrm{M} 1$ of cats. In the more elaborated sensory cortex of the cat, parallel processing streams from each sensory area may target the same general region of a downstream structure, while targeting separate neurons within that region, accounting for our limited ability to detect S1-S2 convergence compared to S1-M1 projections.

A related observation from our current dataset reveals that $\mathrm{S} 1$ and $\mathrm{S} 2$ forepaw projections were distributed into three distinct clusters in the ventrobasal thalamus (specifically VPL). As shown in Fig. 2 and Fig. 7, a core region of VPL contained almost exclusively S1 labeling, while a shell region located more dorsally in VPL contained both S1 and S2 labeled terminal inputs and retrogradely labeled neurons. A third area on the very dorsal edge of VPL contained almost exclusively S2 connections. These findings nicely align with previous dual-retrograde tracing experiments from S1 and S2 forelimb in cats (Spreafico et al. 1981). When analyzed with high resolution using our overlap analysis, these thalamic projections 
from S1 and S2 yielded very weak overlap despite occupying similar regional domains in VPL and having overlapping receptive fields of the forepaw. In contrast, S1-M1 overlap in thalamus was equally as weak as S1-S2, despite significantly robust S1-M1 overlap in striatum and pons. Together these data indicate that (1) S1-M1 integration is critical feature within the feline striatum and pons, though not in thalamus and (2) S1 and S2 may represent parallel processing streams in each of these subcortical structures.

\section{Technical limitations}

In our current study we observed different patterns in sensorimotor convergence which varied from rodents. Primarily, in cats, we saw greater S1-M1 convergence, whereas in rodents we observed greater S1-S2 convergence. Our findings here have two main caveats that limit making overly definitive interpretations about their functional significance. First, whereas we injected the forepaw representation in cats, our previous tracing studies largely targeted the whisker representations in rodents. These body parts have very different behavioral roles and thus may have evolved unique neural circuit organization to facilitate their specific needs. Second, the distance between these cortical representations varies between the two species. In rodents, the whisker regions of S1 and S2 are spatially adjacent, whereas $\mathrm{M} 1$ is many millimeters anterior. Thus, the higher S1-S2 convergence in striatum and pons may be due to a rule of "cortical proximity". We specifically chose the forepaw representation in cats, as the M1, S1 and S2 representations are equidistant, to mitigate effects of cortical proximity. In rodents, however, the M1 and S1 forepaw representations are immediately adjacent with S2 significantly separated, which inherently prevents an equivalent comparison of rodents to cats. Nonetheless, while more mapping studies for different body regions in other species could reveal additional support for variations in cortico-subcortico convergence patterns across different evolutionary lineages, our results indicate distinct differences in the rules that govern sensorimotor corticofugal projection patterns in rodents and cats.

\section{Declarations}

\section{Declarations}

\section{Funding}

This work was supported by National Institute of Health grant NS37532 awarded to Kevin D. Alloway.

\section{Conflicts of interest/Competing interests}

The authors declare that they have no financial interests. Jared B. Smith is currently a paid employee of REGENXBIO, Inc. a for-profit company that has no claims or interest in this research. Shubhodeep Chakrabarti is currently a paid employee of MathWorks, a for-profit company that has no claims or interest in this research.

\section{Ethics approval}


Neuronal tracing experiments were performed on adult felines and all procedures complied with NIH guidelines and were approved by the Penn State Institutional Animal Care and Use Committee (IACUC). Procedures were performed under the guidance of veterinary staff in the Department of Comparative Medicine at the Penn State College of Medicine.

\section{Consent to participate}

Not applicable.

\section{Consent for publication}

Not applicable.

\section{Availability of data and material}

The datasets generated during and/or analyzed during the current study are available from the corresponding author on reasonable request.

\section{Code availability}

Not applicable.

\section{Authors' contributions}

All authors contributed to the study conception and design. Material preparation, data collection and analysis were performed by Jared B. Smith, Shubhodeep Chakrabarti, Todd M. Mowery, Kevin D. Alloway. The first draft of the manuscript was written by Jared B. Smith and all authors commented on previous versions of the manuscript. All authors read and approved the final manuscript.

\section{References}

Alexander GE, DeLong MR, Strick PL (1986) Parallel organization of functionally segregated circuits linking basal ganglia and cortex. Annu Rev Neurosci 9:357-381

Alexander GE, Crutcher MD, DeLong MR (1990) Basal ganglia- thalamocortical circuits: parallel substrates for motor, oculomotor, "prefrontal" and "limbic" functions. Prog Brain Res 85:119-146

Alitto HJ, Usrey WM (2003) Corticothalamic feedback and sensory processing, Curr Opin Neurobiol 13:440-445

Alloway KD (2008) Information processing streams in rodent barrel cortex: the differential functions of barrel and septal circuits. Cerebral Cortex 18: 979-989

Alloway KD, Burton H (1985a) Submodality and columnar organization of the second somatic sensory area in cats. Exp Brain Res 61:128-140 
Alloway KD, Burton H (1985b) Homotypical ipsilateral cortical projections between somatosensory areas I and II in the cat. Neuroscience 14:14-35

Alloway KD, Lou L, Nwabueze-Ogbo F, Chakrabarti S (2006) Topography of cortical projections to the dorsolateral neostriatum in rats: multiple overlapping sensorimotor pathways. J Comp Neurol 499: 33-48

Alloway KD, Mutic JJ, Hoffer ZS, Hoover JE (2000) Overlapping corticostriatal projections from the rodent vibrissal representations in primary and secondary somatosensory cortex. J Comp Neurol 426:51-67

Alloway KD, Olson ML, Smith JB (2008) Contralateral corticothalamic projections from MI whisker cortex: potential route for modulating hemispheric interactions. J Comp Neurol 510:100-116

Alloway KD, Smith JB, Mowery TM, Watson GDR (2017) Sensory processing in the dorsolateral striatum: the contribution of thalamostriatal pathways. Front Syst Neurosci 11, 53

Alloway KD, Smith JB, Beauchemin KJ, Olson ML (2009) Bilateral projections from rat MI whisker cortex to the neostriatum, thalamus, and claustrum: forebrain circuits for modulating whisker behavior. J Comp Neurol 515:548-564

Aoki S, Coulon P, Ruigrok TJH (2019) Multizonal cerebellar influence over sensorimotor areas of the rat cerebral cortex. Cereb Cortex 29: 598-614

Aoki S, Smith JB, Li H, Yan X, Igarashi M, Coulon P, Wickens JR, Ruigrok TJH, Jin X (2019) An open cortico-basal ganglia loop allows limbic control over motor output via the nigrothalamic pathway. eLife 8:e49995 https://doi.org/10.7554/eLife.49995

Arce-McShane FI, Ross CF, Takahashi K, Sessle BJ, Hatsopoulos NG (2016) Primary motor and sensory cortical areas communicate via spatiotemporally coordinated networks at multiple frequencies. Proc Nat Acad Sci USA 113:5083-5088.

Battaglia-Mayer A, Caminiti R (2019) Corticocortical systems underlying high-order motor control. J Neurosci 39:4404-4421

Bjaalie JG, Brodal P (1989) Visual pathways to the cerebellum: segregation in the pontine nuclei of terminal fields from different visual cortical areas in the cat. Neuroscience 29:95-107

Borich MR, Brodie SM, Gray WA, lonta S, Boyd LA (2015) Understanding the role of the primary somatosensory cortex: opportunities for rehabilitation. Neuropsycholigia 79:246-255

Briggs F, Usrey WM (2008) Emerging views of corticothalamic function. Curr Opinion Neurobiol 18:403407

Brodal P (1978) Principles of organization of the monkey corticopontine projection. Brain Res 148:214218 
Chakrabarti S, Alloway KD (2006) Differential origin of projections from SI barrel cortex to the whisker representations in SII and MI. J Comp Neurol 498:624-636

Chakrabarti S, Zhang M, Alloway KD (2008) MI neuronal responses to peripheral whisker stimulation: relationship to neuronal activity in SI barrels and septa. J Neurophysiol 100:50-63

Chapin JK, Lin CS (1984) Mapping the body representation in the SI cortex of anesthetized and awake rats. J Comp Neurol 229:199-213

Charpier S, Pidoux M, Mahon S (2020) Converging sensory and motor cortical inputs onto the same striatal neurons: An in vivo intracellular investigation. PLoS ONE 15(2): e0228260.

Chen S, Augustine GJ, Chadderton P (2016) The cerebellum linearly encodes whisker position during voluntary movement eLife 5:e10509

Colechio EM, Alloway KD (2009) Differential topography of the bilateral cortical projections to the whisker and forepaw regions in rat motor cortex. Brain Struct Funct 213:423-439

Doyon J, Penhune V, Ungerleider LG (2003) Distinct contribution of the cortico-striatal and corticocerebellar systems to motor skill learning. Neuropsychologia 41:252-262

Dudman JT, Gerfen CR (2015) The basal ganglia. In: Paxinos G (ed) The Rat Nervous System, 4th edn. Academic Press, San Diego pp 391-440

Fabri M, Burton H (1991) Ipsilateral cortical connections of primary somatic sensory cortex in rats. J Comp Neurol 311:405-424

Felleman DJ, Wall JT, Cusick CG, Kaas JH (1983) The representation of the body surface in S-I of cats. J Neurosci 3:1648-1669

Flaherty AW, Graybiel AM (1991) Corticostriatal transformations in the primate somatosensory system. Projections from physiologically mapped body-part representations. J Neurophysiol 66:1249-1263

Flaherty AW, Graybiel AM (1993) Two input systems for body representations in the primate striatal matrix: experimental evidence in the squirrel monkey. J Neurosci 13:1120-1137

Flaherty AW, Graybiel AM (1995) Motor and somatosensory corticostriatal projection magnifications in the squirrel monkey. J Neurophysiol 74:2638-2648

Friend DM, Kravitz AV (2014) Working together: basal ganglia pathways in action selection. Trends Neurosci 37:301-303

Gao Z, Davis C, Thomas AM, Economo MN, Abrego AM, Svoboda K, De Zeeuw Cl, Li N (2018) A corticocerebellar loop for motor planning. Nature 563:113-116 
Ghosh S, Brinkman C, Porter R (1987) A quantitative study of the distribution of neurons projecting to the precentral motor cortex in the monkey (M. fascicularis). J Comp Neurol 259:424-444

Hall RD, Lindholm EP (1974) Organization of motor and somatosensory neocortex in the albino rat. Brain Res 66:23-38

Hamadjida A, Dea M, Deffeyes J, Quessy S, Dancause N (2016) Parallel cortical networks formed by modular organization of primary motor cortex outputs. Curr Biol 26:1737-1743

Hintiryan H, Foster NN, Bowman I, Bay M, Song MY, Gou L, Yamashita, S, Bienkowski, MS, Zingg B, Zhu M, et al. (2016) The mouse cortico-striatal projectome. Nat Neurosci 19:1100-1114

Hoffer ZS, Alloway KD (2001) Organization of corticostriatal projections from the vibrissal representations in the primary motor and somatosensory cortical areas of rodents. J Comp Neurol 439:87-103

Hoffer ZS, Hoover JE, Alloway KD (2003) Sensorimotor corticocortical projections from rat barrel cortex have an anisotropic organization that facilitates integration of inputs from whiskers in the same row. $J$ Comp Neurol 466:525-544

Hoffer ZS, Arantes HB, Roth RL, Alloway KD (2005) Functional circuits mediating sensorimotor integration: quantitative comparisons of projections from rodent barrel cortex to primary motor cortex, neostriatum, superior colliculus, and the pons. J Comp Neurol 488:82-100

Hooks BM, Papale AE, Paletzki RF, Feroze MW, Eastwood BS, Couey JJ, Winnubst J, Chandrashekar J, Gerfen CR (2018) Topographic precision in sensory and motor corticostriatal projections varies across cell type and cortical area. Nat Commun 9,3549

Hoover JE, Hoffer ZS, Alloway KD (2003) Projections from primary somatosensory cortex to the neostriatum: the role of somatotopic continuity in corticostriatal convergence. J Neurophysiol 89:15761587

Hoover JE, Strick PL (1993) Multiple output channels in the basal ganglia. Science 259:819-821

Huerta MF, Pons TP (1990) Primary motor cortex receives input from area 3a in macaques. Brain Res 537:367-371

Hunnicutt BJ, Jongbloets BC, Birdsong WT, Gertz KJ, Zhong H, Mao T. (2016) A comprehensive excitatory input map of the striatum reveals novel functional organization. eLife 5:e19103. doi: 10.7554/eLife.19103

Izraeli R, Porter LL (1995) Vibrissal motor cortex in the rat: connections with the barrel field. Exp Brain Res 104:41-54 
Jackson J, Smith JB, Lee AK (2020) The anatomy and physiology of claustrum-cortex interactions. Ann Rev Neurosci 43:231-247

Jones SEG, Coulter JD, Hendry SHC (1978) Intracortical connectivity of architectonic fields in the somatic sensory, motor and parietal cortex of monkeys. J Comp Neurol 181:291-347

Kaas JH (2004) Evolution of somatosensory and motor cortex in primates. Anatomical Record 281:11481156

Khateb M, Schiller J, Schiller Y (2017) Feedforward motor information enhances somatosensory responses and sharpens angular tuning of rat S1 barrel cortex neurons. eLife 6, e21843

Kincaid AE, Wilson CJ (1996) Corticostriatal innervation of the patch and matrix in the rat neostriatum. J Comp Neurol 374:578-592

Klaus A, da Silva JA, Costa RM (2019) What, if, and when to move: basal ganglia circuits and self-paced action initiation. Ann Rev Neurosci 42:459-483

Krubitzer, L (2009) In search of a unifying theory of complex brain evolution. Ann N Y Acad Sci 1156:4467

Kunzle $\mathrm{H}$ (1975) Bilateral projections from precentral motor cortex to the putamen and other parts of the basal ganglia. An autoradiographic study in Macaca fascicularis. Brain Res 88:195-209

Kunzle H (1977) Projections from the primary somatosensory cortex to basal ganglia and thalamus in the monkey. Exp Brain Res 30:481-492

Lee S, Carvell GE, Simons DJ (2008) Motor modulation of afferent somatosensory circuits. Nat Neurosci $11: 1430-1438$

Lee S, Kruglikov I, Huang ZJ, Fishell G, Rudy B (2013) A disinhibitory circuit mediates motor integration in the somatosensory cortex. Nat Neurosci 16:1662-1670

Lee CR, Yonk AJ, Wiskerke J, Paradiso KG, Tepper JM, Margolis DJ (2019) Opposing influence of sensory and motor cortical input on striatal circuitry and choice behavior. Curr Biol 29:1313-1323

Leergaard TB, Alloway KD, Mutic JJ, Bjaalie JG (2000a) Three- dimensional topography of corticopontine projections from rat barrel cortex: correlations with corticostriatal organization. J Neurosci 20:8474-8484

Leergaard TB, Alloway KD, Pham TA, Bolstad I, Hoffer Z, Pettersen C, Bjaalie JG (2004) Three-dimensional topography of corticopontine pro- jections from rat sensorimotor cortex: comparisons with corticostriatal projections reveal diverse integrative organization. J Comp Neurol 478:306-322

Leergaard TB, Lyngstad KA, Thompson JH, Taeymans S, Vos BP, De Schutter E, Bower JM, Bjaalie JG (2000b) Rat somatosensory cerebro- pontocerebellar pathways: spatial relationships of the somatotopic 
map of the primary somatosensory cortex are preserved in a three- dimensional clustered pontine map. $\mathrm{J}$ Comp Neurol 422:246-266

Legg CR, Mercier B, Glickstein M (1989) Corticopontine projection in the rat: the distribution of labeled cortical cells after large injections of horseradish peroxidase in the pontine nuclei. J Comp Neurol 286:427- 441

LeVay S, Sherk H (1981) The visual claustrum of the cat. I. Structure and connections. J Neurosci 1:956980

Levesque M, Charara A, Gagnon S, Parent A, Deschenes M (1996) Corticostriatal projections from layer V cells in rat are collaterals of long-range corticofugal axons. Brain Res 709:311-315

Lu SM, Lin RC (1993) Thalamic afferents of the rat barrel cortex: a light- and electron-microscopic study using Phaseolus vulgaris leucoagglutinin as an anterograde tracer. Somatosens Mot Res 10:1-16

Macchi G, Bentivoglio M, Minciacchi D, Molinari M (1981) The organization of the claustroneocortical projections in the cat studied by means of the HRP retrograde axonal transport. J Comp Neurol 195:681695

Mao T, Kusefoglu D, Hooks BM, Huber D, Petreanu L, Svoboda K (2011) Long-range neuronal circuits underlying the interaction between sensory and motor cortex. Neuron 72:111-123

Matyas F, Sreenivasan V, Marbach F, Waconge C, Barsy B, Mateo C, Aronoff R, Petersen CH (2010) Motor control by sensory cortex. Science 330:1240-1243

Mercier BE, Legg CR, Glickstein M (1990) Basal ganglia and cerebellum receive different somatosensory information in rats. Proc Natl Acad Sci U S A 87:4388-4392

Middleton FA, Strick PL (2000) Basal ganglia and cerebellar loops: motor and cognitive circuits. Brain Res Rev 31:236-250

Mo C, Sherman SM (2019) A sensorimotor pathway via higher-order thalamus. J Neurosci 39:692-704 Mori A (1997) Cortico-cortical connections from somatosensory areas to the motor area of the cortex following peripheral nerve lesion in the cat. Neuroreport 8:3723-3726

Murphy WJ, Pevzner PA, O'Brien SJ (2004) Mammalian phylogenomics comes of age. Trends Genet 20:631-639

Murthy VN, Fetz EE (1992) Coherent 25- to 35-Hz oscillations in the sensorimotor cortex of awake behaving monkeys. Proc Natl Acad Sci USA 89(12):5670-5674

Oh SW, Harris JA, Ng L, Winslow B, Cain N, Mihalas S, Wang Q, et al. (2014) A mesoscale connectome of the mouse brain. Nature 508:207-214 
Pais-Vieira M, Lebedev MA, Wiest MC, Nicolelis MAL (2013) Simultaneous top-down modulation of the primary somatosensory cortex and thalamic nuclei during active tactile discrimination. J Neurosci $33: 4076-4093$

Parent A, Hazrati LN (1995) Functional anatomy of the basal ganglia. I. The cortico-basal gangliathalamo-cortical loop. Brain Res Rev 20:91-127

Proville RD, Spolidoro M, Guyon N, Dugue GP, Selimi F, Isope P, Popa D, Lena C (2014) Cerebellum involvement in cortical sensorimotor circuits for the control of voluntary movements. Nat Neurosci $17: 1233-1239$

Ramanathan S, Hanley JJ, Deniau JM, Bolam JP (2002) Synaptic convergence of motor and somatosensory cortical afferents onto GABAergic interneurons in the rat striatum. J Neurosci 22:81588169

Ramnani N (2006) The primate cortico-cerebellar system: anatomy and function Nat Rev Neurosci 7:511522

Redgrave P, Prescott TJ, Gurney K (1999) The basal ganglia: A vertebrate solution to the selection problem? Neuroscience, 89:1009-1023

Royce GJ (1982) Laminar origin of cortical neurons which project upon the caudate nucleus: A horseradish peroxidase investigation in the cat. J Comp Neurol 205:8-29

Schmahmann JD, Pandya DN (1997) The cerebrocerebellar system. Int Rev Neurobiol 41:31-60

Schwarz C, Mock M (2001) Spatial arrangement of cerebro-pontine terminals. J Comp Neurol 435:418432

Schwarz C, Thier P (1995) Modular organization of the pontine nuclei: dendritic fields of identified pontine projection neurons in the rat respect the borders of cortical afferent fields. J Neurosci 15:34753489

Sherk H (1986) The claustrum and the cerebral cortex. In E. G. Jones \& A. Peters (Eds.), Cerebral cortex: Sensory-motor areas and aspects of cortical connectivity. New York, NY: Plenum

Smith JB, Alloway KD (2010) Functional specificity of claustrum connections in the rat: interhemispheric communication between specific parts of motor cortex. J Neurosci 30:16832-16844

Smith JB, Alloway KD (2013) Rat whisker motor cortex is subdivided into sensory-input and motor-output areas. Front Neural Circuits 7:4

Smith JB, Alloway KD (2014) Interhemispheric claustral circuits coordinate sensory and motor cortical areas that regulate exploratory behaviors. Front Syst Neurosci 8:93 
Smith JB, Alloway KD, Hof PR, Orman R, Reser DH, Watakabe A, et al. (2019a) The relationship between the claustrum and endopiriform nucleus: a perspective towards consensus on cross-species homology. $J$ Comp Neurol 527:476-499

Smith JB, Radhakrishnan H, Alloway KD (2012) Rat claustrum coordinates but does not integrate somatosensory and motor cortical information. J Neurosci 32:8583-8588

Smith JB, Watson GDR, Liang Z, Liu Y, Zhang N, Alloway KD (2019b) A role for the claustrum in salience processing? Front Neuroanat 13:64 DOI: 10.3389/fnana.2019.00064

Spreafico R, Hayes NL, Rustioni A (1981) Thalamic projections to the primary and secondary somatosensory cortices in cat: Single and double retrograde tracer studies. J Comp Neurol 203:67-90

Stepniewska I, Pirkle S, Roy T, Kaas JH (2020) Functionally matched domains in parietal-frontal cortex of monkeys project to overlapping regions of the striatum. Prog Neurobiol (in press)

Stepniewska I, Preuss TM, Kaas JH (1993) Architectonics, somatotopic organization, and ipsilateral cortical connections of the primary motor area (M1) of owl monkeys. J Comp Neurol 330:238-271

Suter BA, Shepherd GMG (2015) Reciprocal Interareal Connections to Corticospinal Neurons in Mouse M1 and S2. J Neurosci 35:2959-2974

Suzuki L, Coulon P, Sabel-Goedknegt, Ruigrok TJH (2012) Organization of cerebral projections to identified cerebellar zones in the posterior cerebellum of the rat. J Neurosci 32:10854-10869

Umeda T, Isa T, Nishimura Y (2019) The somatosensory cortex receives information about motor output. Science Advances 5,7 eaaw5388

Vogt BA, Pandya DN (1977) Cortico-cortical connections of somatic sensory cortex (areas 3, 1 and 2) in the rhesus monkey. J Comp Neurol 177:179-191

Wiesendanger R, Wiesendanger M (1982a) The corticopontine system in the rat. I. Mapping of corticopontine neurons. J Comp Neurol 208:215- 226

Wiesendanger R, Wiesendanger M (1982b) The corticopontine system in the rat. II. The projection pattern. J Comp Neurol 208:227-238

Wiesendanger R, Wiesendanger M, Ruegg DG (1979) An anatomical investigation of the corticopontine projection in the primate (Macaca fascicularis and Saimiri sciureus) - II. The projection from frontal and parental association areas. Neuroscience 4:747-765

Zagha E, Casale AE, Sachdev RNS, McGinley MJ, McCormick DA (2013) Motor cortex feedback influences sensory processing by modulating network state. Neuron 79:567-578 


\section{Figures}
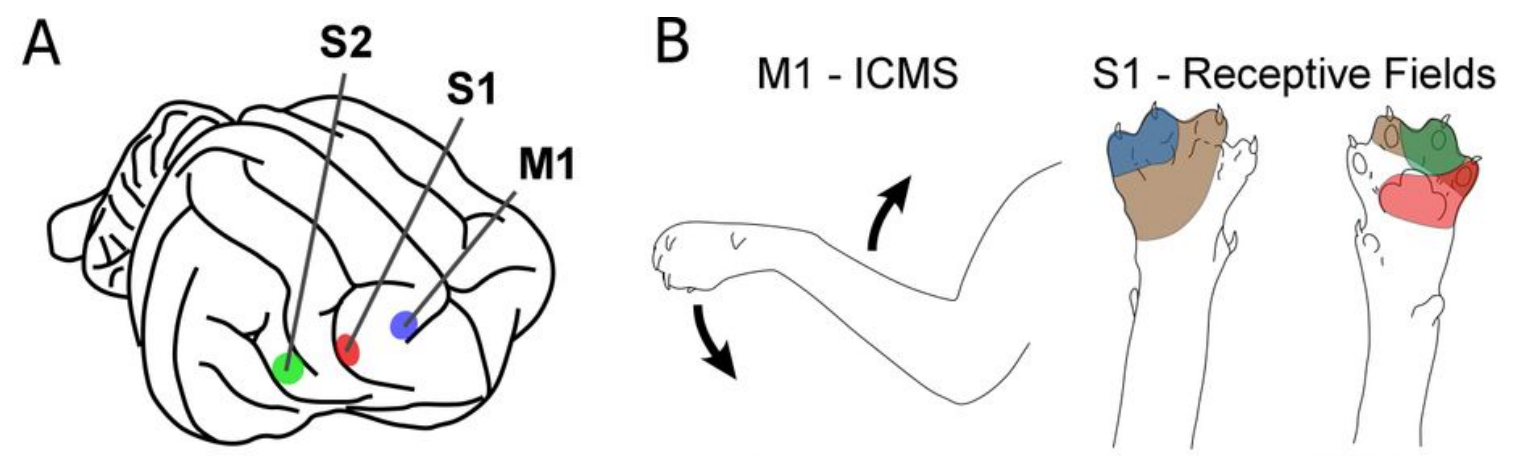

S2 - Receptive Fields
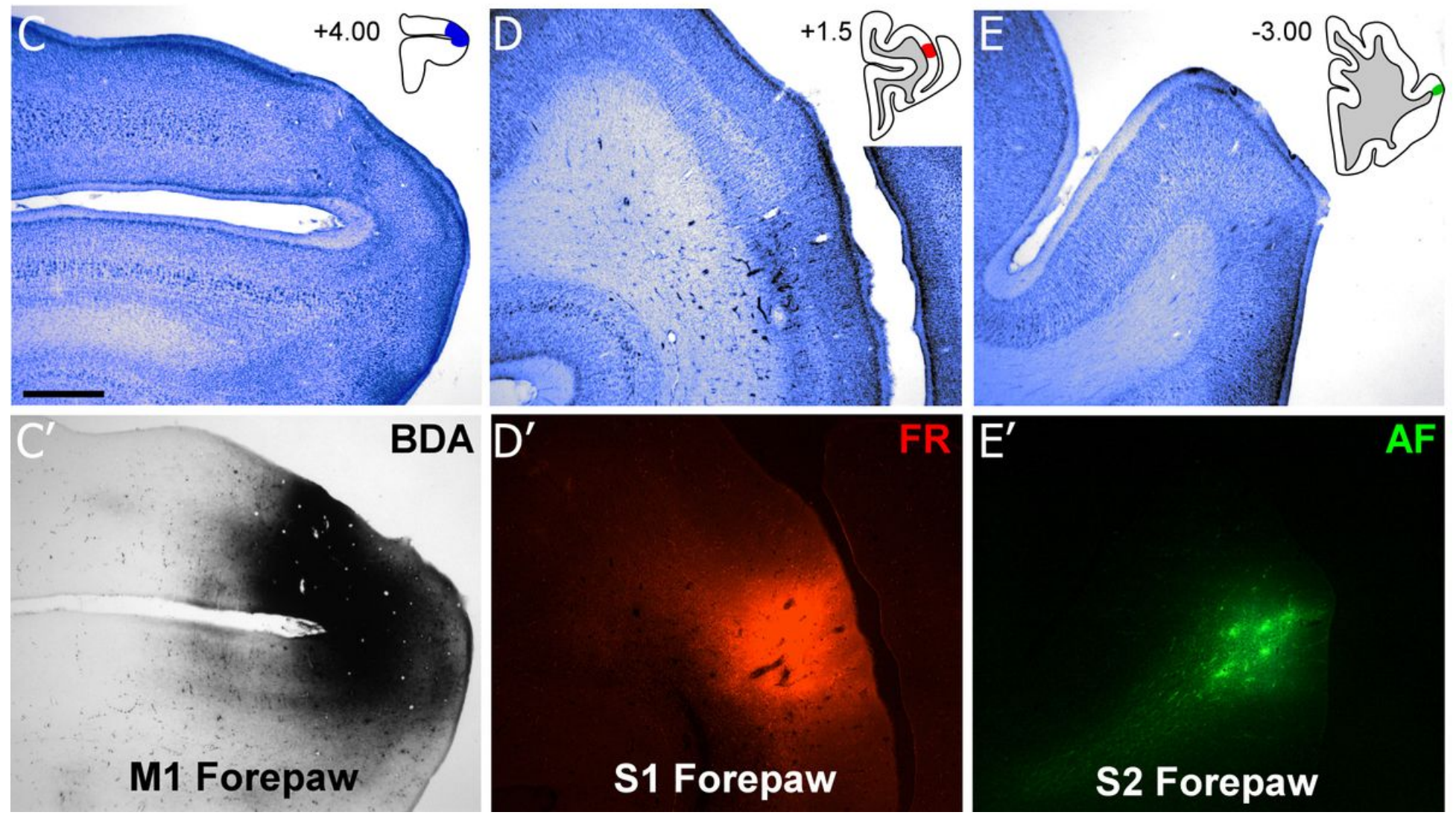

Figure 1

Representative example of cortical mapping and tracer injections from case TI-8. a Diagram illustrating location of tracer injections into the forepaw representations of primary motor cortex (M1), primary somatosensory cortex (S1), and secondary somatosensory cortex (S2). b Diagrams of cortical mapping results, showing the forelimb/forepaw movements from M1 intracortical microstimulation (ICMS) as well as receptive fields in S1 and S2 from case TI-8. Colors correspond to the receptive fields at different cortical sites within S1 and S2. c Nissl-stained coronal section showing location of M1 forepaw in TI-8. Inset shows digital reconstruction of entire coronal section and location of injection sites of biotinylated dextran amine (BDA; injection shown in blue). c' Brightfield photomicrograph showing BDA injection on the adjacent serial section processed with DAB to visualize the tracer (see Methods). d,d' Nissl (d) and fluorescent ( $d^{\prime}$ ) photomicrographs showing the location of Fluororuby (FR) injections into S1 forepaw. e,e' 
Photomicrographs of Alexa-fluor 488 (AF) injections into the S2 forepaw. Coordinates relative to bregma. Scale bars: $1 \mathrm{~mm}$ in c.

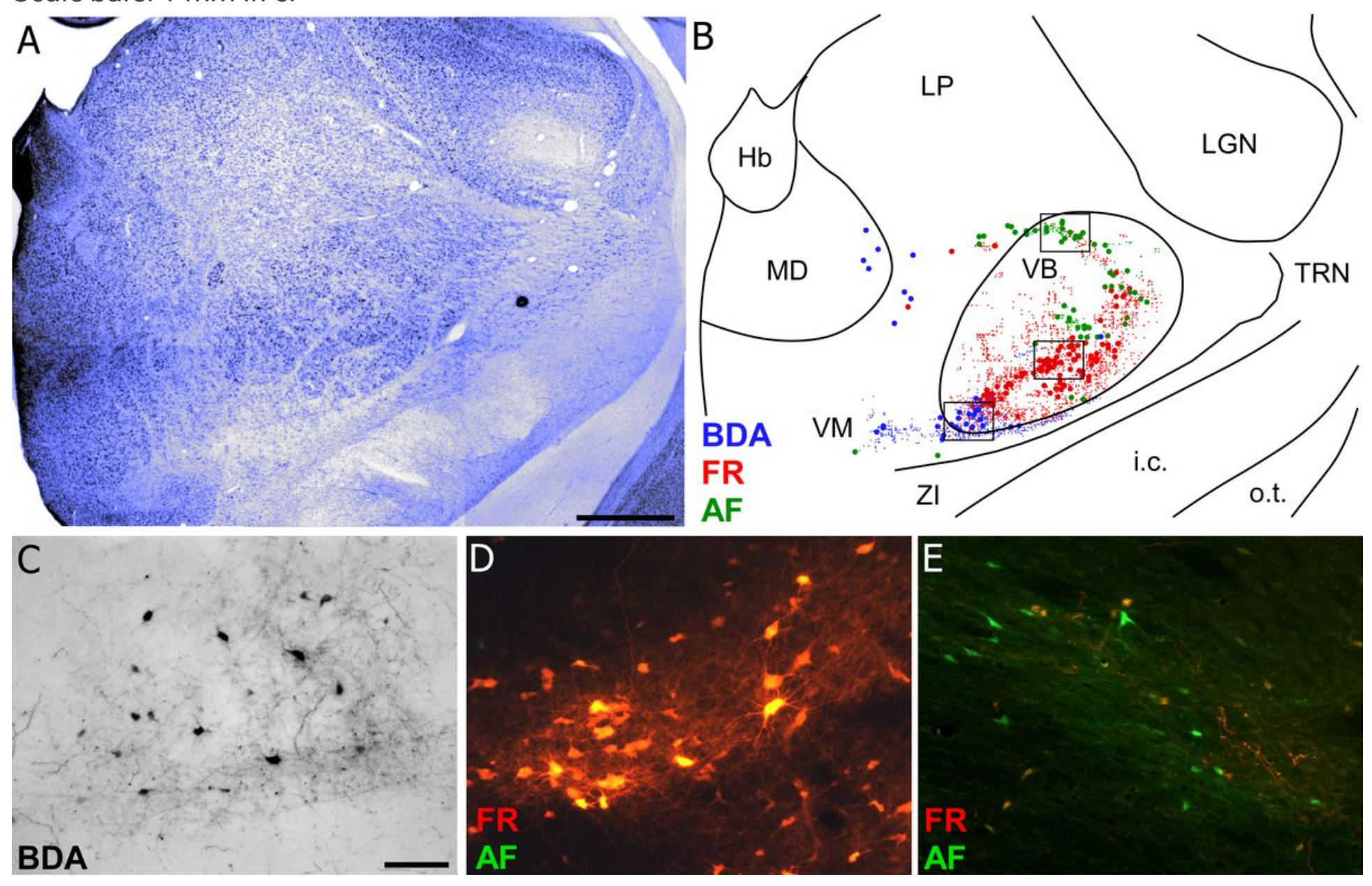

\section{Figure 2}

Representative example of tracer labeling in the thalamus of case TI-8 (same case as injection sites shown in Fig. 1). a Nissl-stained coronal section of thalamus. b Digital reconstruction showing labeling of all three tracers in thalamus. Small dots represent anterogradely-labeled synaptic terminal boutons; large dots represent retrogradely-labeled neuronal cell bodies. Colors correspond to different tracers (BDA, blue; $F R$, red; AF, green). c-e Photomicrographs of BDA labeling in thalamus resulting from the M1 injection (c), FR-labeling in thalamus from the S1 injection (d), and AF-labeling from the S2 injection (e). Photomicrographs correspond to the lower-left (c), middle (d), and upper-right (e) insets in panel b, respectively. Abbreviations: $\mathrm{Hb}$, habenula; i.c., internal capsule; LP, lateral posterior nucleus; LGN, lateral geniculate nucleus; MD, mediodorsal nucleus; o.t., optic tract; TRN, thalamic reticular nucleus; VB, ventrobasal complex; VM, ventromedian nucleus; ZI, zona incerta. Scale bars: $1 \mathrm{~mm}$ in a, $250 \mathrm{~mm}$ in c. 


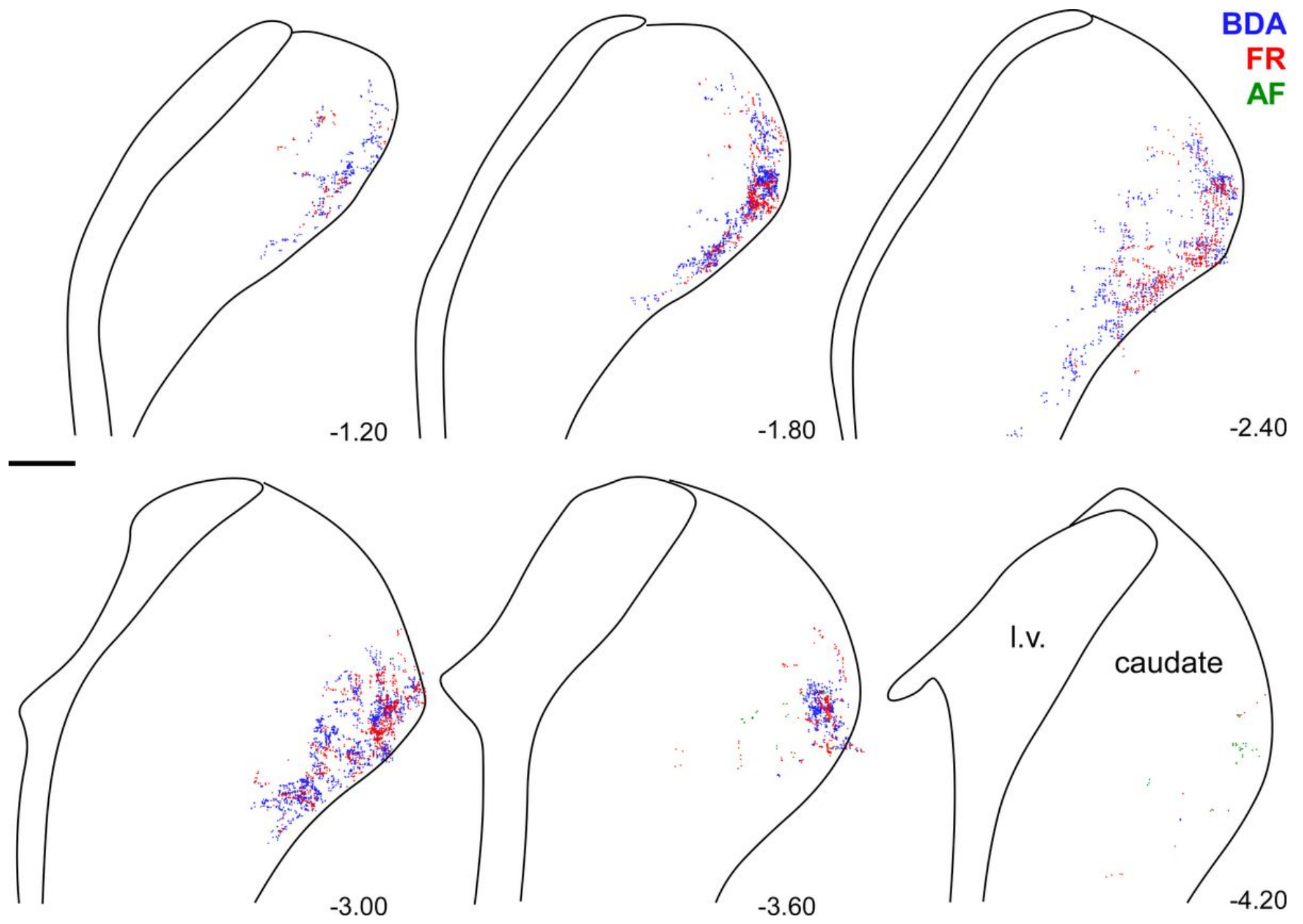

Figure 3

Representative digital reconstruction of terminal labeling in striatum (caudate nucleus) of case TI-8. Colors correspond to different tracers (BDA, blue; FR, red; AF, green). Note the dense intermingling of the M1 (blue) and S1 (red) projections in the dorsolateral region. Coordinates relative to bregma. Scale bar: 1 $\mathrm{mm}$. 

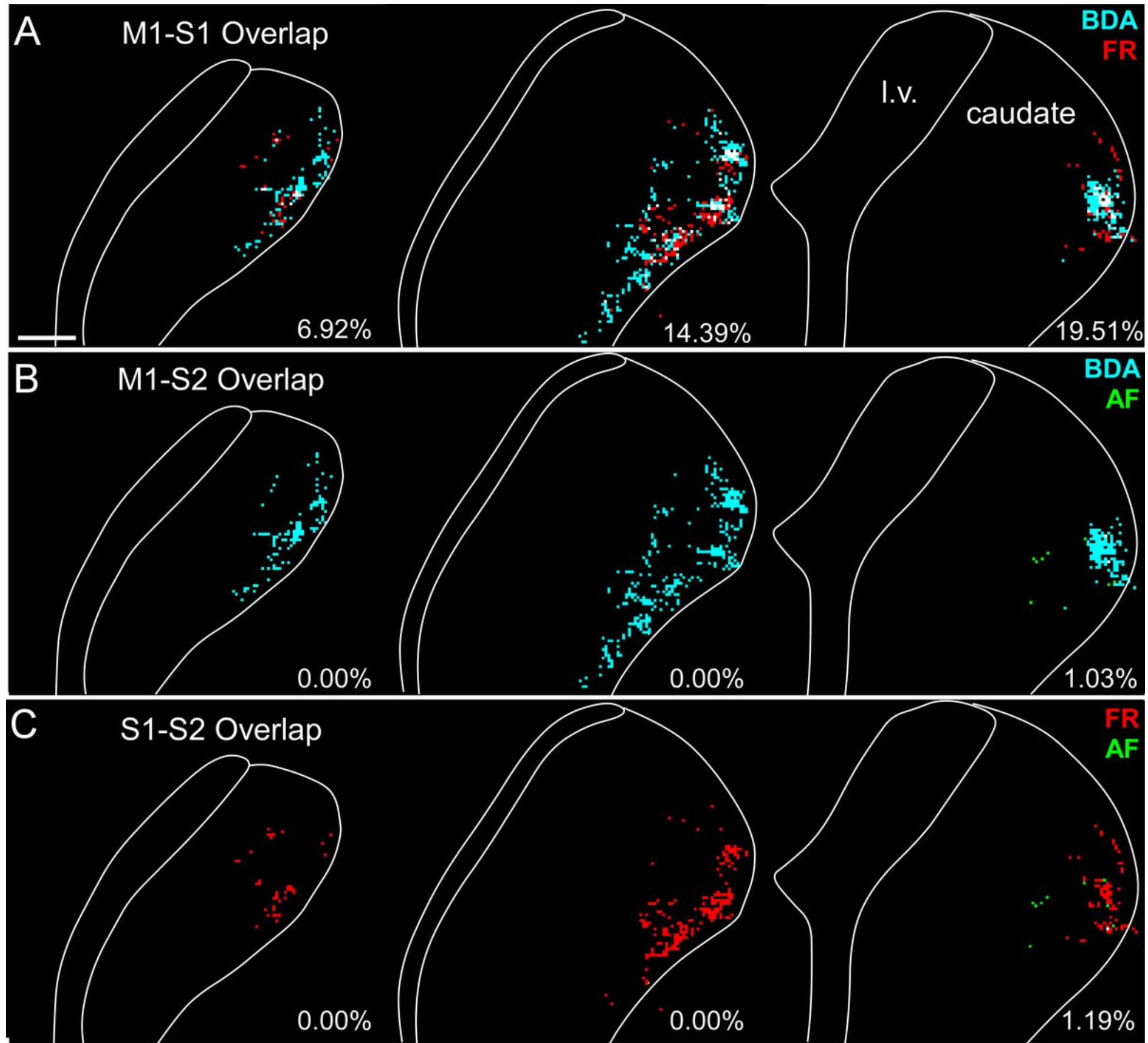

Figure 4

Representative overlap analysis of anterograde tracer labeling in striatum (caudate nucleus) from case TI-8. Colors correspond to different tracers (BDA, blue; FR, red; AF, green). White bins represent overlap. Bin size $=50 \mathrm{~mm} 2$. Each panel shows only two tracers, with M1 (blue) and S1 (red) in panel a, M1 (blue) and S2 (green) in b, and S1 (red) and S2 (green) in panel c. Percentages represent proportion of total bins containing overlap. Note the moderate overlap of M1 and S1 projections in the dorsolateral region shown in panel a. Scale bars: $1 \mathrm{~mm}$ in a. 

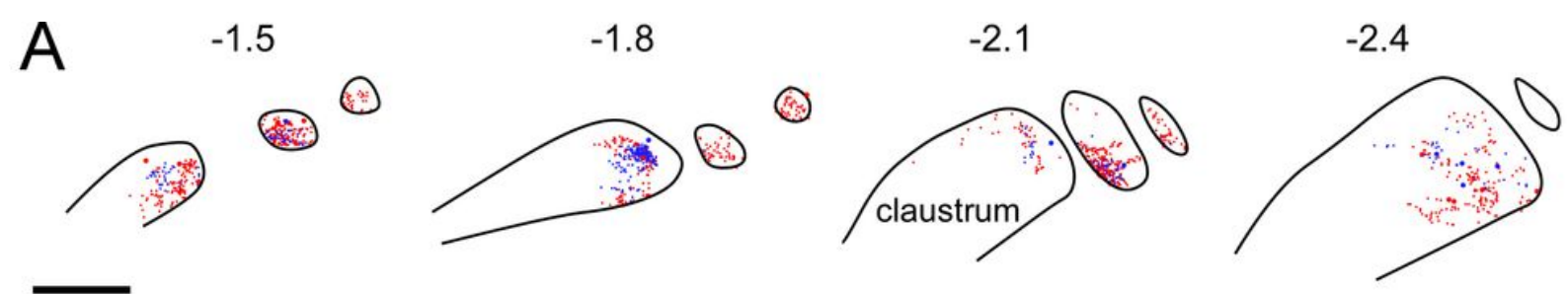

B

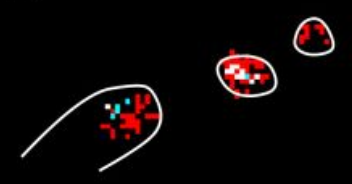

$11.76 \%$

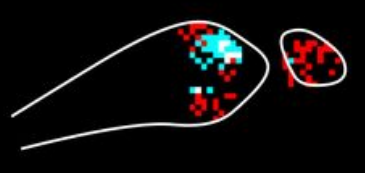

$7.21 \%$

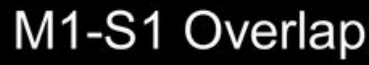

(1)

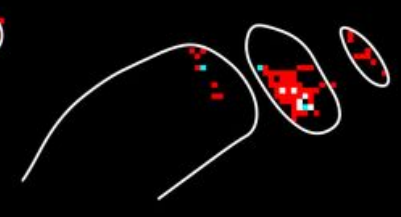

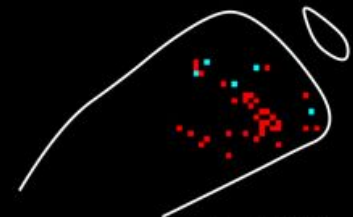

$0.00 \%$

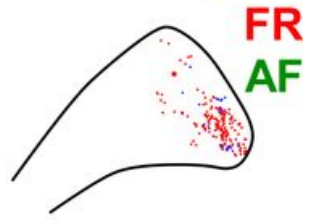

BDA

FR

C

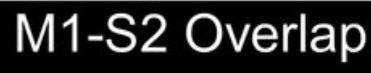

$0.00 \%$
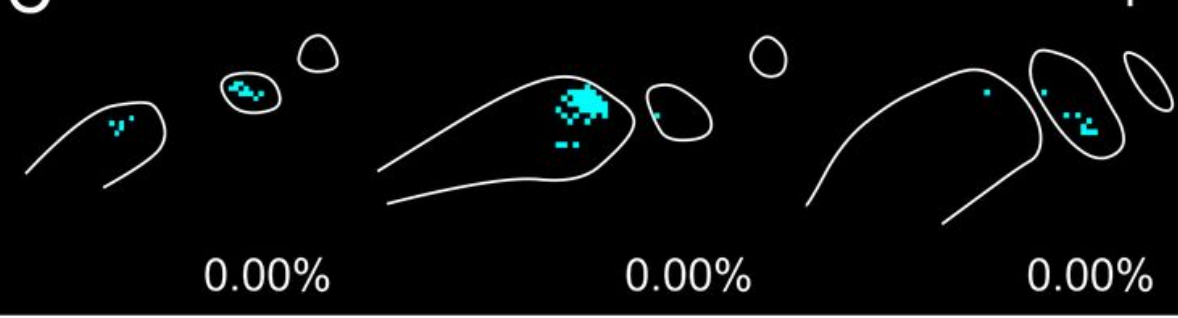

$0.00 \%$

S1-S2 Overlap

D
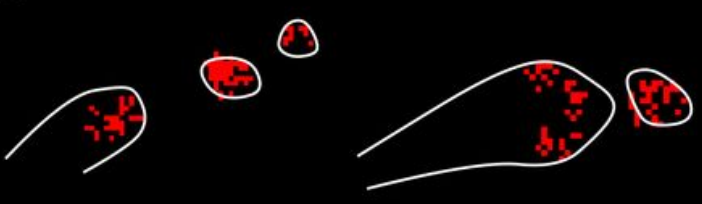

(1)

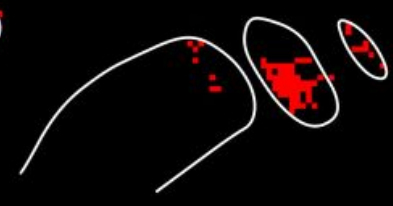

$0.00 \%$

$0.00 \%$

$0.00 \%$
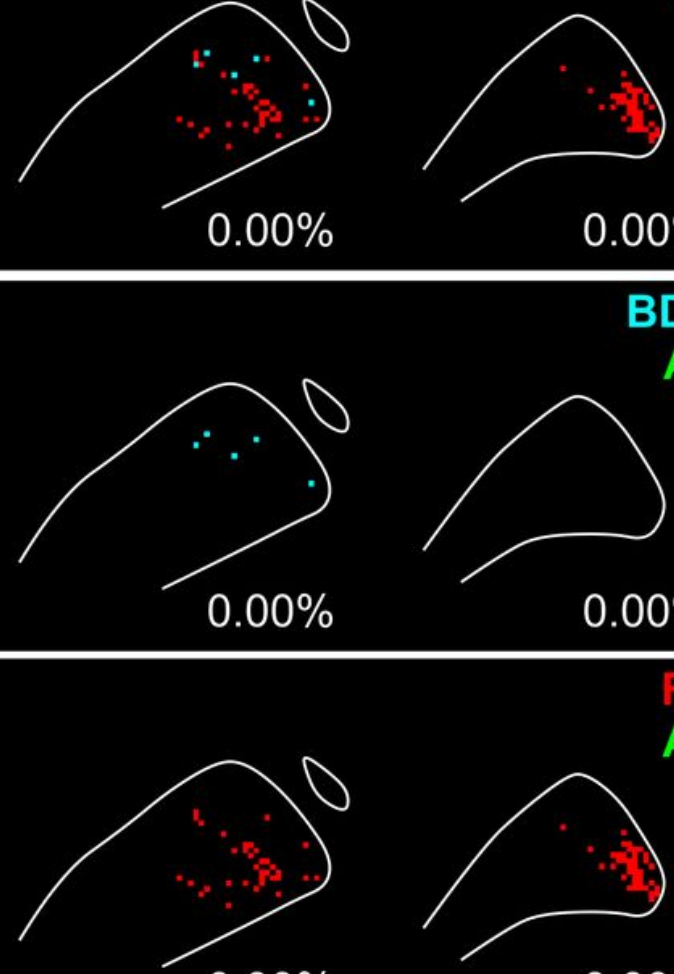

BDA

AF

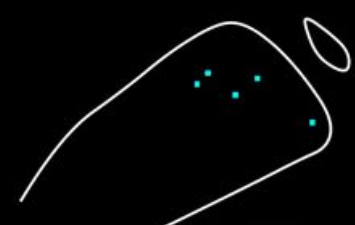

$0.00 \%$

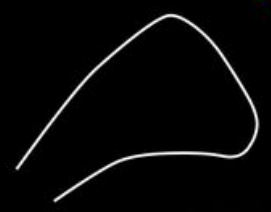

$0.00 \%$

\section{Figure 5}

Representative digital reconstruction of tracer labeling and overlap analysis of anterogradely labeled terminals in claustrum of case TI-8. (a) Digital reconstruction of tracer labeling throughout anteriorposterior extent of claustrum. Colors correspond to different tracers (BDA, blue; FR, red; AF, green). Small dots represent terminal synaptic boutons, large dots represent neuronal cell bodies. Note the strong presence of S1 projections. Coordinates relative to bregma. Scale bar: $1 \mathrm{~mm}$. (b-d) Overlap analysis of anterogradely labeled terminals. Colors correspond to different tracers (BDA, blue; FR, red; AF, green). White bins represent overlap. Bin size $=50 \mathrm{~mm} 2$. Each panel shows only two tracers, with M1 (blue) and S1 (red) in panel b, M1 (blue) and S2 (green) in c, and S1 (red) and S2 (green) in panel d. Percentages represent proportion of total bins containing overlap. Note the moderate overlap of M1 and S1 projections shown in panel b. Scale bars: $1 \mathrm{~mm}$ in a. 

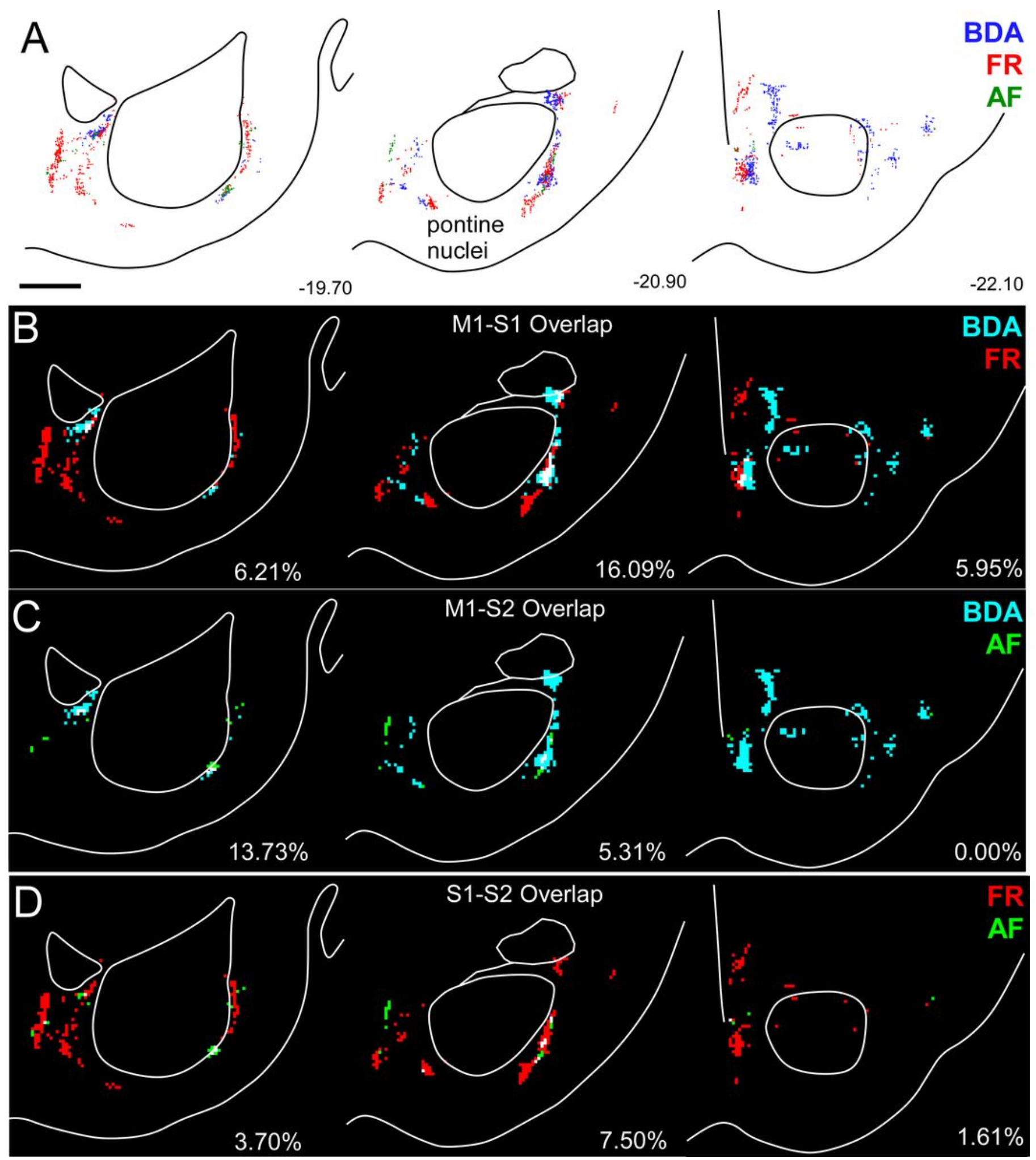

\section{Figure 6}

Representative digital reconstruction of tracer labeling and overlap analysis of anterogradely labeled terminals in the pontine nuclei of case TI-8. (a) Digital reconstruction of tracer labeling throughout anterior-posterior extent of the pons. Colors correspond to different tracers (BDA, blue; FR, red; AF, green). Small dots represent terminal synaptic boutons. Note the strong intermingling of M1, S1, and S2 projections. Coordinates relative to bregma. (b-d) Overlap analysis of anterogradely labeled terminals. 
Colors correspond to different tracers (BDA, blue; FR, red; AF, green). White bins represent overlap. Bin size = 50mm2. Each panel shows only two tracers, with M1 (blue) and S1 (red) in panel b, M1 (blue) and S2 (green) in c, and S1 (red) and S2 (green) in panel d. Percentages represent proportion of total bins containing overlap. Note the moderate overlap of all tracers. Scale bar: $1 \mathrm{~mm}$ in a.
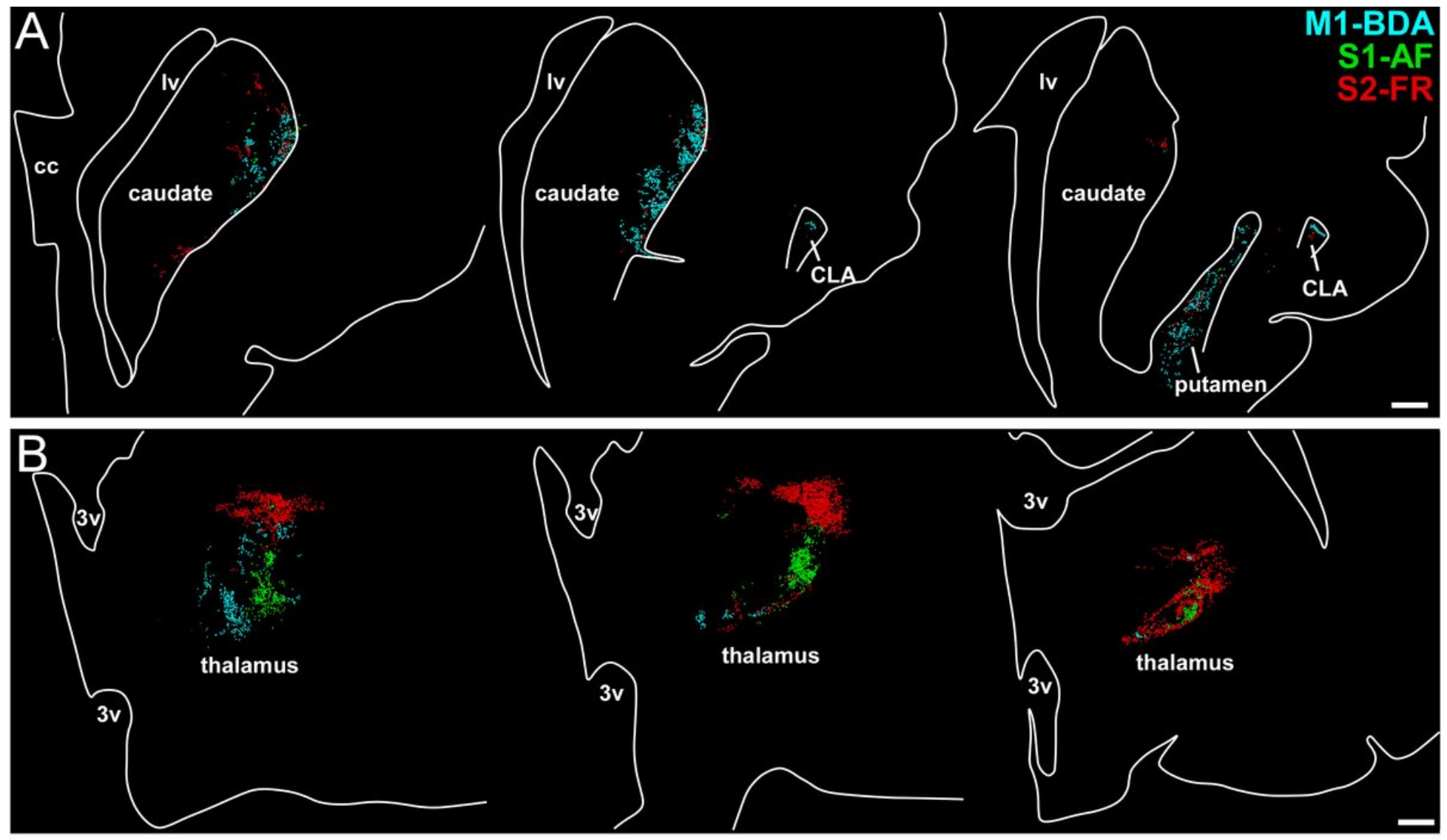

C

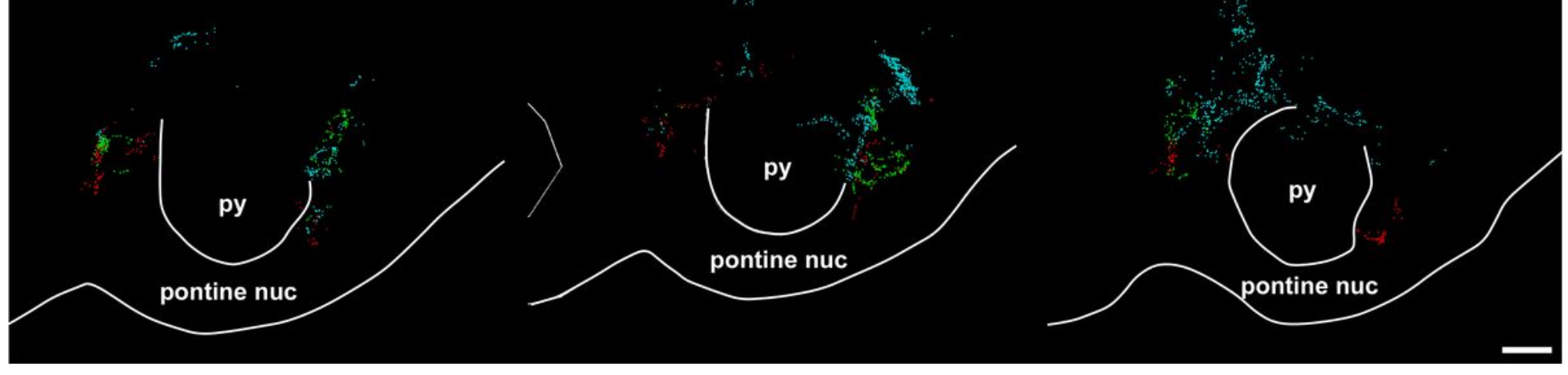

\section{Figure 7}

Representative digital reconstruction of terminal labeling in the striatum and claustrum (a), thalamus (b), and pontine nuclei (c) of case TI-9. Note that in this case tracers were assigned to different cortical areas: M1-BDA (blue), S1-AF (green), S2 FR (red). Scale bar: $1 \mathrm{~mm}$ in a and b, 500mm in c. 

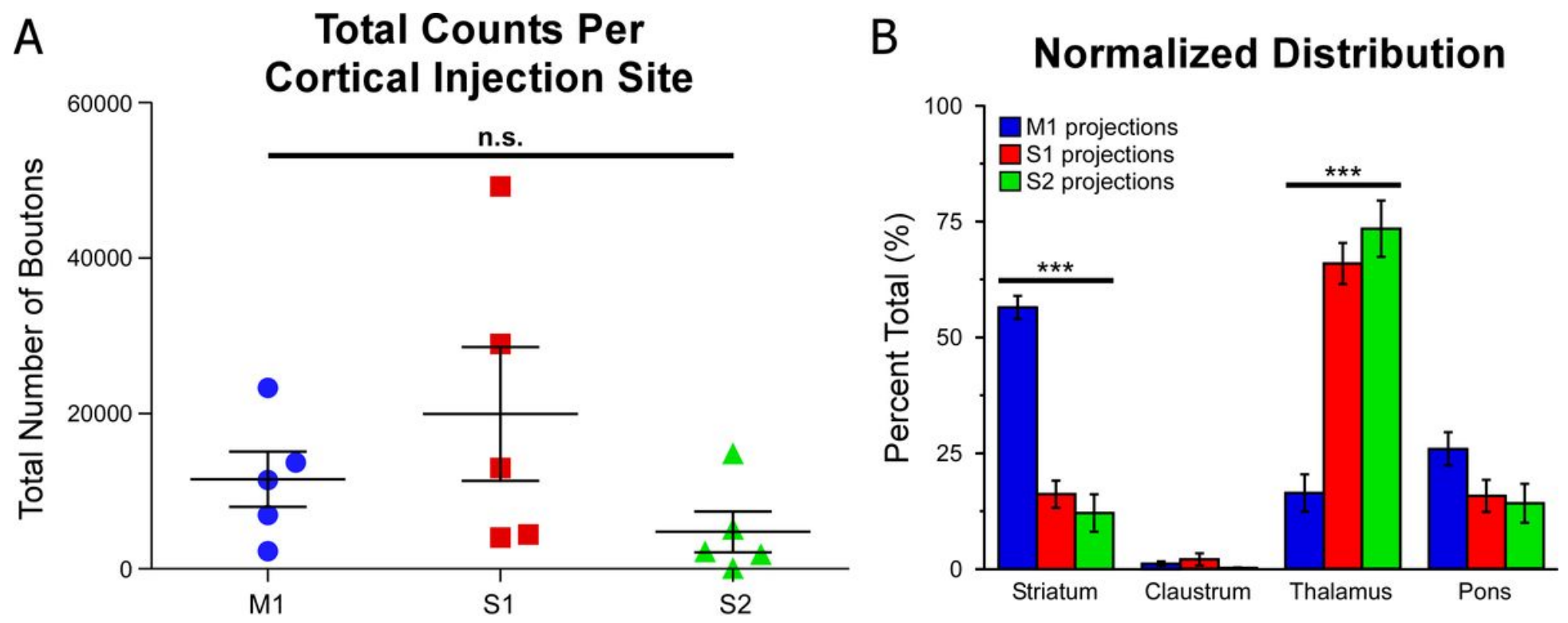

Figure 8

Quantification of anterograde terminal labeling across all animals included in the final analysis $(n=5)$. a Analysis of total number of terminal synaptic boutons counted in the striatum, claustrum, thalamus and pons for each cortical area injected across all animals included in the final analysis (not significant, 1way ANOVA, $F=1.85, p=0.20$ ). b Normalized distribution of anterograde terminal bouton labeling across all subcortical regions for each cortical area injected (i.e. all M1 bars add up to $100 \%$, all S1 bars add up to $100 \%$, etc.). Statistical analysis showed a significant effect (2-way ANOVA, interaction $F=43.24$, $\mathrm{p}<0.0001)$. Post-hoc Bonferroni comparisons showed M1 projections to striatum were significantly stronger than $S 1(t=8.11, p<0.001)$ and $S 2(t=8.93, p<0.001)$, whereas in thalamus $M 1$ projections were significantly weaker than $S 1(t=9.96, p<0.001)$ and $S 2(t=11.47, p<0.001)$. 
A Cat Sensorimotor Cortex B Tracer Injections

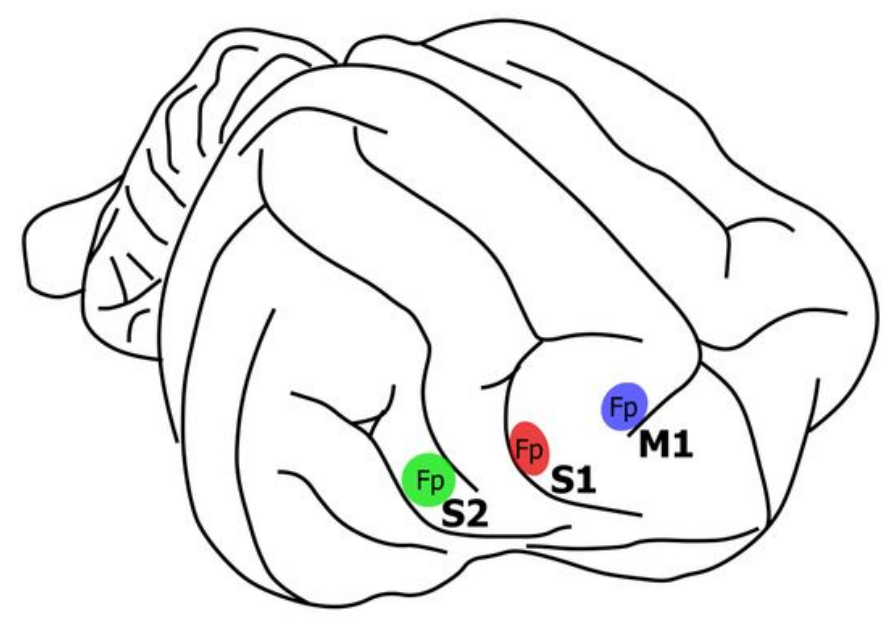

\section{Rat Sensorimotor Cortex Tracer Injections}

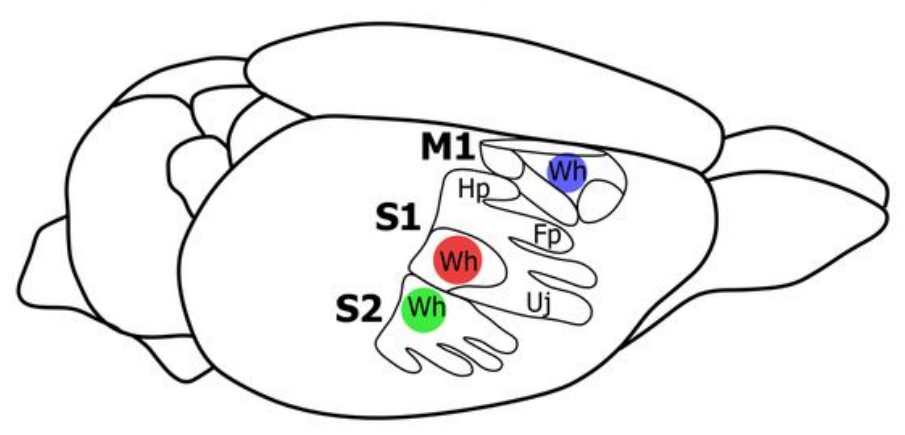

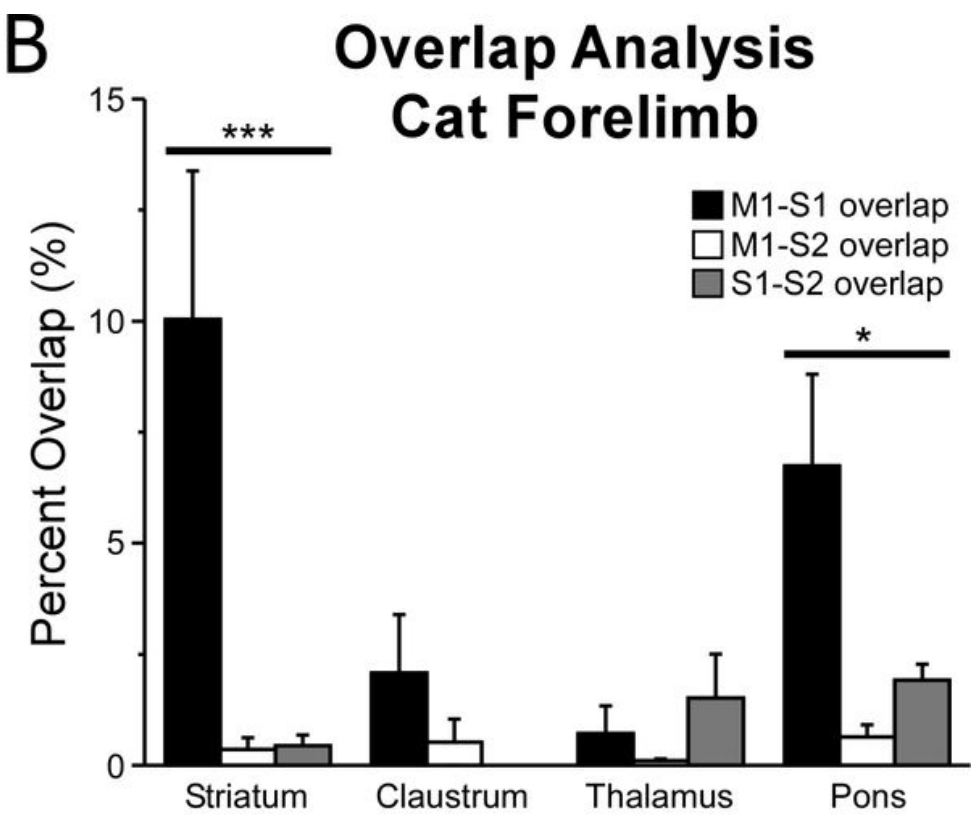

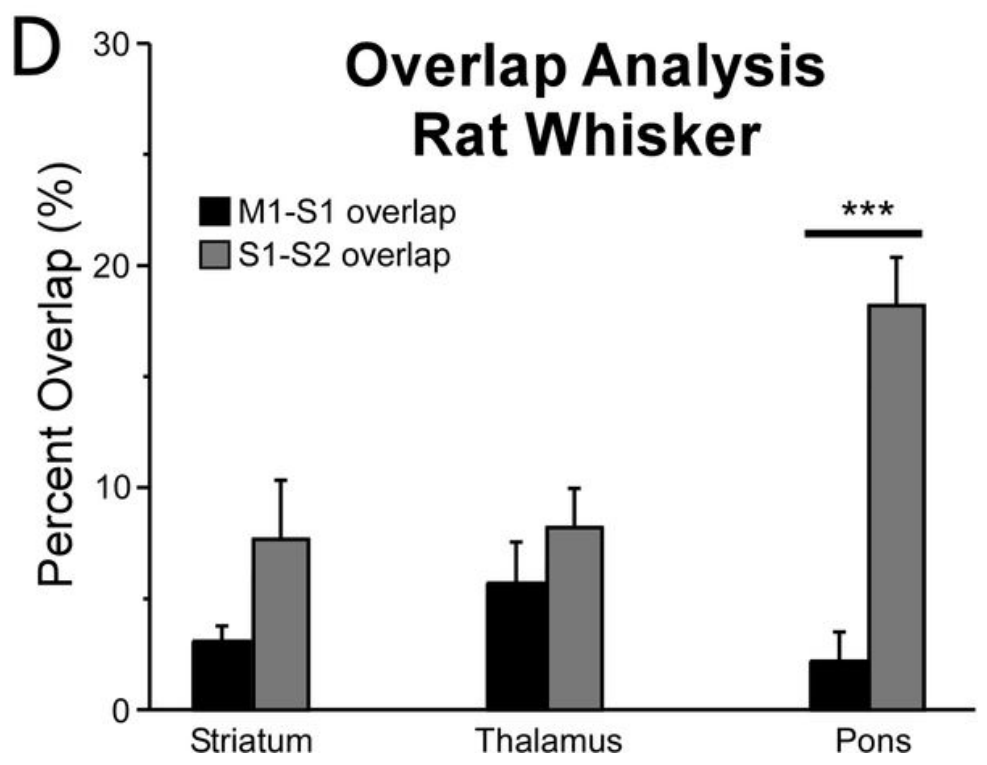

Figure 9

Quantification of anterograde tracer overlap from sensorimotor cortex injections in cat and rat. a Injection scheme from this study employing a triple tracer paradigm injecting $M 1, S 1$, and S2 in the same animal with three different tracers. b Summary of overlap analysis using $50 \mathrm{~mm} 2$ bins, with a threshold requiring two counts per bin revealed a significant effect (2-way ANOVA, interaction $F=4.00, p<0.01$ ). Post-hoc Bonferroni tests revealed that M1-S1 overlap was significantly greater than M1-S2 and S1-S2 in the striatum ( $t=5.43, p<0.001$ and $t=5.38, p<0.001$; respectively) and in the pons $(t=3.44, p<0.05$ and $t=2.70$, n.s.; respectively). c Injection scheme showing location of anterograde tracer injections in rodents for previous studies (see Table 1 in Leergaard et al., 2004 for information about injections and Figure 10 for overlap data). d Graph summarizing findings from overlap analysis (bin size $50 \mathrm{~mm} 2$ ) in rodent tracing studies shows more S1-S2 overlap than M1-S1, that was significant in the pons $(t=4.87, p<0.001)$. 